\title{
Off-diagonal cosmological solutions in emergent gravity theories and Grigory Perelman entropy for geometric flows
}

\author{
Sergiu I. Vacaru ${ }^{1,2, a}$, Elşen Veli Veliev ${ }^{3, b}$, Laurenţiu Bubuianu ${ }^{4,5, c}$ \\ ${ }^{1}$ Physics Department, California State University at Fresno, Fresno, CA 93740, USA \\ ${ }^{2}$ Department of Theoretical Physics and Computer Modelling, 101 Storozhynetska Street, Chernivtsi 58029, Ukraine \\ ${ }^{3}$ Department of Physics, Kocaeli University, 41380 Izmit, Turkey \\ ${ }^{4}$ SRTV-Studioul TVR Iaşi, 28 Alexandru Lapuşneanu Street, 700057 Iasi, Romania \\ ${ }^{5}$ University Apollonia, 2 Muzicii Street, 700399 Iasi, Romania
}

Received: 1 November 2020 / Accepted: 23 December 2020 / Published online: 25 January 2021

(C) The Author(s) 2021

\begin{abstract}
We develop an approach to the theory of relativistic geometric flows and emergent gravity defined by entropy functionals and related statistical thermodynamics models. Nonholonomic deformations of G. Perelman's functionals and related entropic values used for deriving relativistic geometric evolution flow equations. For self-similar configurations, such equations describe generalized Ricci solitons defining modified Einstein equations. We analyse possible connections between relativistic models of nonholonomic Ricci flows and emergent modified gravity theories. We prove that corresponding systems of nonlinear partial differential equations, PDEs, for entropic flows and modified gravity posses certain general decoupling and integration properties. There are constructed new classes of exact and parametric solutions for nonstationary configurations and locally anisotropic cosmological metrics in modified gravity theories and general relativity. Such solutions describe scenarios of nonlinear geometric evolution and gravitational and matter field dynamics with pattern-forming and quasiperiodic structure and various space quasicrystal and deformed spacetime crystal models. We analyse new classes of generic off-diagonal solutions for entropic gravity theories and show how such solutions can be used for explaining structure formation in modern cosmology. Finally, we speculate why the approaches with Perelman-Lyapunov type functionals are more general or complementary to the constructions elaborated using the concept of Bekenstein-Hawking entropy.
\end{abstract}

\footnotetext{
a e-mail: sergiu.vacaru@gmail.com;

sergiuvacaru@mail.fresnostate.edu (corresponding author)

b e-mails: elsen@kocaeli.edu.tr; elsenveli@hotmail.com

${ }^{\mathrm{c}}$ e-mail: laurentiu.bubuianu@tvr.ro
}

\section{Contents}

1 Introduction ................ 2

2 Spacetime $2+2$ and $3+1$ fibrations with elastic and quasiperiodic structures . . . . . . . . . . 4

2.1 Nonlinear connections with $2+2$ splitting of Lorentz manifolds . . . . . . . . . . . . . . 4

2.2 Nonholonomic $3+1$ splitting adapted to $2+2$ structures .............. 6

2.3 Quasiperiodic space and time QC configurations 7 2.3.1 1-d relativistic time QC structures . . . 7

2.3.2 3-d QC structures on curved spaces . . . 7

2.4 Distributions defining spacetime elastic configurations . . . . . . . . . . . . 7

3 Relativistic geometric flows and modified entropic gravity 8

3.1 Modified spacetime and hypersurface Perelman's functionals . . . . . . . . . . . . . 9

3.1.1 Generalized Perelman functionals for entropic geometric flows and MGTs . . . . . . . . 9

3.1.2 Nonholonomic 3-d space like hypersurface $\mathrm{F}$ - and $\mathrm{W}$-functionals . . . . . . . 10

3.2 Geometric flow equations for modified gravitational and matter fields . . . . . . . . . . 10

3.3 Entropic gravity and gravitational field equations as Ricci solitons . . . . . . . . . . . . . . 11

4 Decoupling and integrability of entropic flow equations 11

4.1 Geometric flows with parametric modified Einstein equations . . . . . . . . 11

4.1.1 Entropic quasiperiodic flow modifications of gravitational field equations . . . . . 11

4.1.2 Effective entropic sources for stationary and/or cosmological configurations . . . 11

4.2 Nontrivial Ricci d-tensors and decoupling of entropic flow equations . . . . . . . . 12 
4.2.1 Off-diagonal metric ansatz, (non) holonomic variables, and ODEs and PDEs . . 12

4.2.2 Cosmological Ricci d-tensors, LC-conditions, and nonlinear symmetries . . . . . . 13

4.3 Integrability of entropic quasiperiodic geometric flow equations . . . . . . . . . . . . . 14

4.3.1 Off-diagonal cosmological solutions with elastic quasiperiodic structures . . . . . . 14

4.3.2 Quadratic line elements for off-diagonal cosmological configurations with elastic flows . . 15

4.3.3 Off-diagonal Levi-Civita entropic and quasiperiodic cosmological configurations . . . . . 16

5 Entropic quasiperiodic flows and cosmological solutions . 16

5.1 The AFDM for entropic flow cosmological solutions ................ . . 16

5.2 Nonlinear PDEs for entropic quasiperiodic cosmology ............... . 17

5.2.1 Cosmological solutions for entropic quasiperiodic sources . . . . . . . . . . . 17

5.2.2 Cosmological configurations with nonstationary entropic generating functions . . 18

5.2.3 Emergent quasiperiodic cosmology from both generating functionals and sources . 19

5.3 Cosmological metrics evolving in entropic quasiperiodic media . . . . . . . . . . . . . 19

5.3.1 Cosmological evolutions generated by nonstationary entropic sources . . . . . . 20

5.3.2 Cosmology from nonstationary entropic generating functions ....... . 20

5.3.3 Cosmological configurations for entropic sources and generating functions . . . . . 21

6 Conclusions and discussion . . . . . . . . . . 21

References ................ 23

\section{Introduction}

The most inspiring ideas in recent development of the gravity theory and cosmology are on the emergent thermodynamic nature of the spacetime geometry, when the Einstein equations can be derived using area-entropy formulas for horizons of black holes, BH, [1-3] and from supposed elastic properties of gravity [5-7]. A substantial progress includes the research on the microscopic origin of Bekenstein-Hawking entropy in string theory [8], the holographic principle [9, 10], $\mathrm{BH}$ complementarity [11], and gauge/gravity correspondence $[12,13]$. Here we note a subsequent development of the (anti) de Sitter, (A)dS, and conformal field theories, CFTs, and AdS/CFT, correspondence [14] and formulating laws of the thermodynamics of 'apparent' horizons [15].

Later, it was proposed that gravity theories and generalized/modified/linearized Einstein equations are consequences of the quantum entanglement connecting nearby spacetime regions [16-21]. Recent theoretical activities are devoted to proofs that the entanglement first law and dual gravity are derived from the CFT and/or reveal a deep connection to ideas on emergence of spacetime and gravity from general quantum information principles [4,7,22-25]. It was pointed out that in a model of dual gravity with entanglement is equivalent to the full (nonlinear) equations [26]. An intriguing conjecture that gravity links to an entropic force as a spacetime elasticity was proposed by Verlinde [6,7]. It was based on the idea that gravitational interactions result from information regarding the positions of material bodies. Emergent phenomena for gravity were investigated by many other authors and their efforts involve the holographic principle in particle physics and the information theory. There were studied geometric models and possible applications related to gravity and quantum computers; quantum gravity; cosmological inflation and acceleration; and dark energy and dark matter physics etc., see [27-31] and references therein.

In the quest to explore the connection between the models of emergent gravity and in modified gravity theories, MGTs, or general relativity, GR, one involve a strict area law for the BH, (A)dS, or entanglement entropy and further developments for holographic models. To derive gravitational field equations was considered that a small but nonzero volume law entropy would compete with, and at large distances involves, the area law. In certain models this is due to thermalization, elastic spacetime properties, quantum entanglement, holographic effects etc. It was proposed that such a phenomenon occurs in the dS space being responsible for the presence of a cosmological horizon. Nevertheless, in this series of two works, see [32] as a partner paper with complementary results, we deal with quite different issues on geometric flow modifications of gravity and spacetime thermodynamics. It is just shown that relativistic generalizations with a corresponding choice of evolution and thermodynamic functionals support the ideas on the origin of gravity as an effect of the entropic force but with a new type of geometric thermodynamics entropy. Such an approach with Perelman-Lyapunov entropy type functionals was developed in our works on entropic nonholonomic geometric flow evolution, nonlinear dynamics and thermodynamics for relativistic, noncommutative, fractional, supersymmetric, Finsler-Lagrange-Hamilton etc. generalizations of the theory of Ricci flow evolution and applications in modern gravity and cosmology [33-41].

The goal of this paper is to elaborate on geometric and physical theories relating relativistic generalizations of the Poincaré-Thurston conjecture, ${ }^{1}$ emergent and modified

\footnotetext{
${ }^{1}$ Originally formulated and proved, respectively, due to R. Hamilton and G. Perelman, for Ricci flows of Riemannian metrics; in certain sense, this states that our universe has the topology of a three dimensional sphere, which is considered as one of the fundamental results in modern mathematics.
} 
gravity constructions, and Verlinde conjecture that gravity results from an entropic force as a spacetime elasticity which explain fundamental properties of dark matter, DM, and dark energy, DE, in modern cosmology [6,7]. On topology and geometry of Ricci flows, we refer to classical works [42-45] and reviews of mathematical results in monographs [46-48]). Here we note that Friedan published a series of works on nonlinear sigma models, $\sigma$-models, in $2+\varepsilon$ dimensions, see [49-51] where geometric flow equations were introduced for the renorm group, RG, theories, see recent results in [52-54].

The other goal of this article is to develop the anholonomic frame deformation method, AFDM, (on early works see [55-57] and references therein), for constructing exact and parametric quasiperiodic solutions of geometric entropic flow and modified gravity equations. For reviews of recent results on black hole solutions in MGTs [58,59], space and time like (quasi) crystals, pattern forming and nonlinear gravitational wave structures and applications in modern cosmology see [60-64] and references therein. We elaborate on new classes of generic off-diagonal stationary and cosmological solutions with entropic geometric flows which for self similar Ricci soliton configurations result in equations considered in Verlinde works [6,7] and a covariant generalization due to Hossenfelder [65], see also critics Refs. [66,67].

Three lines of evidence motivate this article and the partner letter [32]. First, we use our former results and nonholonomic geometric methods [33-41] that generalized/modified relativistic flow equations and Einstein equations in GR and MGTs can be derived as systems of PDEs for modified Ricci solitons for respective nonholonomic modifications of G. Perelman's F- and W-entropy functionals. On modified gravity and applications in modern cosmology and astrophysics, see reviews [63,68-70]. Second, a number of recent works invoke ideas on origin of gravity as an emergent effect of the entropic force, entanglement etc., see [4,6,7,1626]. We argue that this can be grounded and explained, at a deeper level, through modifications of the PoincaréThurston conjecture on geometric flows, when the F- and Wfunctionals are generalized for metrics and generalized connections on Lorentz manifolds and/or certain supersymmetric/noncommutative/fractional/stochastic generalizations. In our approach, the spacetime evolution and gravity are treated via geometric entropy values which allows to formulate respective statistical thermodynamics models. Third, new advanced methods for constructing exact solutions in MGTs and GR allows us to proceed directly toward definition of gravitational entropy and thermodynamic values making no use of holography, area-entropy relation, CFT duality etc. Due to the competition between area and volume law of generalized W-entropy, we can characterize thermodynamically new classes of BH and cosmological solutions with quasiperiodic structure, locally anisotropic inflation and acceler- ating scenarios, exhibiting memory effects in the form of an entropy displacement caused by matter etc.

This article is organized as follows: In Sect. 2, we provide an introduction to the geometry of double, $2+2$ and $3+1$ dimensional, spacetime fibrations defining elastic and quasiperiodic configurations both for gravitational and (effective) matter fields. Main concepts and most important results on nonlinear connection geometry on nonholonomic Lorentz manifolds, hypersurface geometric objects, and modified emergent/elastic gravity theories are outlined. There are studied the geometry of distributions, and respective Lagrange densities and geometric evolution or dynamical fields defining elastic and quasiperiodic structures.

Section 3 is devoted to the theory of geometric flows and modified entropic gravity. We postulate canonical nonholonomic deformations of Perelman's F- and W-functionals encoding geometric flow evolution scenarios of entropic spacetimes with quasiperiodic structure. Such values are defined in relativistic 4-d form and for 3-d hypersurface projections. There are derived respective (generalized R. Hamilton) geometric flow equations for entropic quasiperiodic flows. The concept of nonholonomic Ricci solitons as self-similar configurations is elaborated and related modifications of the Einstein equations are analyzed. We speculate on connection between relativistic generalizations of the Poincaré-Thurston conjecture for Ricci flows and geometric proofs of E. Verlinde's conjecture.

In Sect. 4, we develop and apply the anholonomic frame deformation method, AFDM, [32,38,40,58,63,64] in order to prove general decoupling properties and integrability of nonholonomic geometric flow and Ricci soliton equations encoding elastic and quasiperiodic spacetime and (effective) matter fields properties. Such solutions are described by generic off-diagonal metrics, and generalized connections, depending on all spacetime coordinates and temperature like parameters via general classes of generating functions and (effective) sources of entropic gravity and matter fields.

Then, in Sect. 5, we consider the AFDM for constructing cosmological solutions for entropic quasiperiodic flow and MGTs. We emphasize that there are certain nonlinear symmetries relate possible classes of generating functions and (effective) sources all encoding entropic, quasiperiodic, pattern forming, space and time quasicrystal, solitonic and other type structures. There are studied cosmological configurations generated by entropic quasiperiodic sources, nonstationary generating functions, cosmological metrics evolving in (off-) diagonal elastic and/or quasiperiodic media.

Finally, we conclude our work and discuss certain perspectives of the theory of G. Perelman and E. Verlinde entropic geometric flow and emergent gravity theories in Sect. 6. 


\section{Spacetime $2+2$ and $3+1$ fibrations with elastic and quasiperiodic structures}

In this section, we summarize necessary results on the geometry of Lorentz manifolds enabled with nonholonomic (i.e. non-integrable, equivalently, anholonomic) distributions defining double $2+2$ and $3+1$ fibrations. There are developed nonholonomic geometric methods which are important for elaborating theories of relativistic Ricci flows and possible applications in modern cosmology and astrophysics, see details in [38-40]. As explicit examples, we shall consider nonholonomic distributions modelling elastic and/or quaisperiodic space and time structures (for instance, quasicrystal or solitonic like configurations) $[6,7,59,61,62,64,65]$. It should be noted that the $2+2$ nonholonomic splitting is important for proofs of general decoupling and integration properties of the relativistic geometric and entropic flow evolution, nonholonomic Ricci soliton and (entropic modified) Einstein equations, see Sect. 4 . Additional $3+1$ decompositions adapted to $2+2$ splitting will be used for defining and computing entropic and thermodynamic like values for various classes of solutions of physically important systems of nonlinear partial differential equations, PDEs, see Sect. 5.

\subsection{Nonlinear connections with $2+2$ splitting of Lorentz manifolds}

Let us consider a four dimensional, 4-d, Lorentzian manifold $V, \operatorname{dim} V=4$, with local pseudo-Euclidean signature $(+++-)$ for a metric field $\mathbf{g}=(h \mathbf{g}, v \mathbf{g})$. The conventional horizontal, h, and vertical, v, nonholonomic decomposition is defined by a nonlinear connection, $\mathrm{N}$-connection, structure $\mathbf{N}$. Such a geometric object can be always introduced as a Whitney sum

$$
\mathbf{N}: T \mathbf{V}=h \mathbf{V} \oplus v \mathbf{V},
$$

where $T \mathbf{V}$ is the tangent bundle on $\mathbf{V}$. The concept of nonholonomic manifold is used for a manifold enabled with a nonholonomic distribution. In this work, this refers to a Lorentz spacetime $\mathbf{V}:=(V, \mathbf{N})$ enabled with a $\mathrm{N}$-connection structure of type (1). In local coordinates, $\mathbf{N}=N_{i}^{a}(u) d x^{i} \otimes$ $\partial_{a}$, where $N_{i}^{a}$ are $\mathrm{N}$-connection coefficients. ${ }^{2}$ Any set $\left\{N_{i}^{a}\right\}$ defines subclasses of $\mathrm{N}$-linear (co) frames which allows $\mathrm{N}$ -

\footnotetext{
2 We can parameterize the local coordinates in the form $u^{\mu}=\left(x^{i}, y^{a}\right)$, (in brief, $u=(x, y)$ ), where indices respectively take values $i, j, \ldots=$ 1,2 and $a, b, \ldots=3,4$, considering that $u^{4}=y^{4}=t$ is the time like coordinate. The Einstein convention on summation on "up-low" repeating indices will be applied if contrary will not be stated for some special cases. We use boldface symbols for spaces and geometric objects adapted to a $\mathrm{N}$-connection splitting.
}

adapted diadic decompositions of geometric and physical objects. $^{3}$

On any nonholonomic manifold $\mathbf{V}$, we can consider covariant derivatives determined by affine (linear) connections which are, or not, adapted to a $\mathrm{N}$-connection structure. A distinguished connection, $d$-connection, is a linear connection $\mathbf{D}=(h \mathbf{D}, v \mathbf{D})$ which preserves under parallel transport a h-v-decomposition (1). ${ }^{4}$ For any d-connection $\mathbf{D}$, we can define and compute in standard form the d-torsion, $\mathbf{T}$, the nonmetricity, $\mathbf{Q}$, and the d-curvature, $\mathbf{R}$, tensors

$$
\begin{aligned}
\mathbf{T}(\mathbf{X}, \mathbf{Y}) & :=\mathbf{D}_{\mathbf{X}} \mathbf{Y}-\mathbf{D}_{\mathbf{Y}} \mathbf{X}-[\mathbf{X}, \mathbf{Y}], \mathbf{Q}(\mathbf{X}) \\
& :=\mathbf{D}_{\mathbf{X}} \mathbf{g}, \mathbf{R}(\mathbf{X}, \mathbf{Y}):=\mathbf{D}_{\mathbf{X}} \mathbf{D}_{\mathbf{Y}}-\mathbf{D}_{\mathbf{Y}} \mathbf{D}_{\mathbf{X}}-\mathbf{D}_{[\mathbf{X}, \mathbf{Y}]},
\end{aligned}
$$

where $\mathbf{X}$ and $\mathbf{Y}$ are vector fields (i.e. d-vectors) on $T \mathbf{V} .^{5}$

Any metric tensor $\mathbf{g}=(h \mathbf{g}, v \mathbf{g})$, on a nonholonomic $\mathbf{V}$ can be written as a distinguished tensor, d-tensor (d-metric), with respective splitting into $\mathrm{h}$ - and $\mathrm{v}$-indices,

$$
\begin{aligned}
\mathbf{g}= & g_{\alpha}(u) \mathbf{e}^{\alpha} \otimes \mathbf{e}^{\beta}=g_{i}(x) d x^{i} \otimes d x^{i} \\
& +g_{a}(x, y) \mathbf{e}^{a} \otimes \mathbf{e}^{a},
\end{aligned}
$$

where the nonholonomic dual frame structure $\mathbf{e}^{\alpha}$ is chosen in a form when the matrix of metric coefficients $g_{\alpha \beta}$ is considered in diagonal form for $g_{\alpha}:=g_{\alpha \alpha}, g_{i}:=g_{i i}$ and $g_{a}:=g_{a a}$. With respect to a dual local coordinate basis $d u^{\alpha}$ the same metric field is expressed

$$
\begin{aligned}
\mathbf{g}= & \underline{g}_{\alpha \beta} d u^{\alpha} \otimes d u^{\beta}, \\
& \text { where } \underline{g}_{\alpha \beta}=\left[\begin{array}{ll}
g_{i j}+N_{i}^{a} N_{j}^{b} g_{a b} & N_{j}^{e} g_{a e} \\
N_{i}^{e} g_{b e} & g_{a b}
\end{array}\right] .
\end{aligned}
$$

\footnotetext{
${ }^{3}$ Such $\mathrm{N}$-adapted local bases, $\mathbf{e}_{v}=\left(\mathbf{e}_{i}, e_{a}\right)$, and cobases, $\mathbf{e}^{\mu}=$ $\left(e^{i}, \mathbf{e}^{a}\right)$, are defined by formulas

$\mathbf{e}_{i}=\partial / \partial x^{i}-N_{i}^{a}(u) \partial / \partial y^{a}, e_{a}=\partial_{a}$, and

$e^{i}=d x^{i}, \mathbf{e}^{a}=d y^{a}+N_{i}^{a}(u) d x^{i}$
}

and their arbitrary frame/coordinate transforms. The term nonholonomic used for a Lorentz manifold $\mathbf{V}$ comes from the fact that a basis (tetrad, equivalently, vierbeind) $\mathbf{e}_{v}=\left(\mathbf{e}_{i}, e_{a}\right)$ satisfies certain relations $\left[\mathbf{e}_{\alpha}, \mathbf{e}_{\beta}\right]=\mathbf{e}_{\alpha} \mathbf{e}_{\beta}-\mathbf{e}_{\beta} \mathbf{e}_{\alpha}=W_{\alpha \beta}^{\gamma} \mathbf{e}_{\gamma}$, with nontrivial anholonomy coefficients $W_{i a}^{b}=\partial_{a} N_{i}^{b}, W_{j i}^{a}=\Omega_{i j}^{a}=\mathbf{e}_{j}\left(N_{i}^{a}\right)-\mathbf{e}_{i}\left(N_{j}^{a}\right)$. Holonomic (integrable) configurations are obtained if and only if $W_{\alpha \beta}^{\gamma}=0$.

${ }^{4}$ In general, a linear connection $D$ is not adapted to a prescribed $\mathrm{N}$ connection structure, i.e. it is not a d-connection. In such a case, one should be not used a boldface symbols for respective geometric objects determined by $D$.

5 We can compute in $\mathrm{N}$-adapted form the coefficients of any $\mathrm{d}$ connection $\mathbf{D}=\left\{\Gamma_{\alpha \beta}^{\gamma}=\left(L_{j k}^{i}, L_{b k}^{a}, C_{j c}^{i}, C_{b c}^{a}\right)\right\}$. The coefficients of torsion, nonmetricity and curvature d-tensors are parameterized by $h$ - and $v$-indices, respectively, $\mathcal{T}=\left\{\mathbf{T}_{\alpha \beta}^{\gamma}=\right.$ $\left.\left(T_{j k}^{i}, T_{j a}^{i}, T_{j i}^{a}, T_{b i}^{a}, T_{b c}^{a}\right)\right\}, \quad \mathcal{Q}=\left\{\mathbf{Q}_{\alpha \beta}^{\gamma}\right\}, \mathcal{R}=\left\{\mathbf{R}_{\beta \gamma \delta}^{\alpha}=\right.$ $\left.\left(R_{h j k}^{i}, R_{b j k}^{a}, R_{h j a}^{i}, R_{b j a}^{c}, R_{h b a}^{i}, R_{b e a}^{c}\right)\right\}$, when the coefficients formulas for such values determined by using $\Gamma_{\alpha \beta}^{\gamma}$ and their partial derivatives. 
Using frame transforms (in general, not $\mathrm{N}$-adapted), we can transform any metric into a d-metric (2) an off-diagonal form with $\mathrm{N}$-coefficients. For nontrivial anholonomy coefficients, such a metric is generic off-diagonal.

For our geometric constructions, there are two important linear connections determined by the same metric structure:

$\mathbf{g} \rightarrow \begin{cases}\nabla: \nabla \mathbf{g}=0 ;{ }^{\nabla} \mathbf{T}=0, & \text { the Levi-Civita, LC, connection; } \\ \mathbf{D}: \mathbf{D} \mathbf{g}=0 ; h \mathbf{T}=0, v \mathbf{T}=0 . & \text { the canonical d-connection. }\end{cases}$

Here we note that if we prescribe a $\mathrm{N}$-connection structure $\mathbf{N}$, we can define a canonical d-connection $\mathbf{D}$ and compute certain nontrivial torsion coefficients $h v \mathbf{T}$ completely defined by certain off-diagonal coefficients containing $N_{i}^{a}(u)$ in (3) and/or nontrivial anholonomy coefficients $W_{\alpha \beta}^{\gamma}$, see footnote 3 . Of course, we can introduce an infinite number of metric compatible d-connections but not all such connections allow to decouple physically important systems of nonlinear PDEs (in our case, for nonholonomic geometric flows and modified, or Einstein, gravity). A D (4) allows us to prove the decoupling and integration properties of such equations in Sect. 4.

The LC-connection $\nabla$ (4) can be defined uniquely by a metric $\mathbf{g}$ without any $\mathrm{N}$-connection structure but $\nabla$ can be distorted always to a necessary type d-connection allowing a general decoupling and integrability of certain important physically important systems of nonlinear PDEs. In our previous works [38-40] (see there necessary geometric details and $[59,61,62,64]$ for applications of the AFDM), we used a "hat" symbol (like $\widehat{\mathbf{D}}$ ) for the canonical d-connection in (4). In this paper, we shall work only with $\nabla$ and $\mathbf{D}=\widehat{\mathbf{D}}$ and omit "hats" on respective geometric objects. We note that all constructions performed for $\nabla$ and $\mathbf{D}$ are related by a distortion relation, $\mathbf{D}[\mathbf{g}, \mathbf{N}]=\nabla[\mathbf{g}, \mathbf{N}]+\mathbf{Z}[\mathbf{g}, \mathbf{N}]$, where $\mathbf{Z}$ is the distortion tensor determined in standard algebraic form by the torsion tensor $\mathbf{T}$; all values are completely defined by the metric tensor $\mathbf{g}$ adapted to $\mathbf{N} .^{6}$

The Ricci tensors of $\mathbf{D}$ and $\nabla$ are defined and computed in standard forms for different linear connection structures but defined by the same metric tensor by contracting respective indices. We denote them, respectively, $\mathbf{R} i c=\left\{\mathbf{R}_{\alpha \beta}:=\right.$ $\left.\mathbf{R}_{\alpha \beta \gamma}^{\gamma}\right\}$ and Ric $=\left\{R_{\alpha \beta}:=R_{\alpha \beta \gamma}^{\gamma}\right\}$. Any (pseudo) Riemannian geometry can be equivalently described by both geometric data $(\mathbf{g}, \nabla)$ and $(\mathbf{g}, \mathbf{N}, \mathbf{D})$, when the canonical distortion relations $\mathbf{R}={ }^{\nabla} \mathbf{R}+{ }^{\nabla} \mathbf{Z}$ and $\mathbf{R} i c=R i c+Z i c$, with respective distortion d-tensors $\nabla \mathbf{Z}$ and $Z i c$, are computed for the canonical distortion relations $\mathbf{D}=\nabla+\mathbf{Z}$, see details

\footnotetext{
6 The values $h \mathbf{T}$ and $v \mathbf{T}$ are respective torsion components which vanish on conventional h- and v-subspaces, but there are nontrivial components $h v \mathbf{T}$ defined by certain anholonomy (equivalently, nonholonomic/nonintegrable) relations. Such a d-torsion is induced by nonholonomic configurations.
}

in $[35,38-40,63]$ (in those works, there are used different systems of notations).

Using $\mathrm{N}$-adapted coefficients of the canonical Ricci dtensor,

$$
\begin{gathered}
\mathbf{R}_{\alpha \beta}=\left\{\mathbf{R}_{i j}:=\mathbf{R}_{i j k}^{k}, \mathbf{R}_{i a}:=-\mathbf{R}_{i k a}^{k},\right. \\
\left.\mathbf{R}_{a i}:=\mathbf{R}_{a i b}^{b}, \mathbf{R}_{a b}:=\mathbf{R}_{a b c}^{c}\right\},
\end{gathered}
$$

we can compute the scalar of canonical d-curvature, ${ }^{s} \mathbf{R}:=$ $\mathbf{g}^{\alpha \beta} \mathbf{R}_{\alpha \beta}=g^{i j} \mathbf{R}_{i j}+g^{a b} \mathbf{R}_{a b}$. This geometric object is different from the scalar curvature of the LC-connection, $R:=$ $\mathbf{g}^{\alpha \beta} R_{\alpha \beta}$.

Using $\nabla$, the Einstein equations in GR are written in standard form,

$R_{\alpha \beta}-\frac{1}{2} g_{\alpha \beta} R=\varkappa^{m} T_{\alpha \beta}$

In these formulas, ${ }^{m} T_{\alpha \beta}$ is the energy-momentum tensor of matter fields ${ }^{A} \varphi$ determined by a general Lagrangian ${ }^{m} \mathcal{L}\left(\mathbf{g}, \nabla,{ }^{A} \varphi\right)$, where $\varkappa$ is the gravitational coupling constant for GR. ${ }^{7}$

We can define nonholonomic gravitational field equations using the Ricci d-tensor (5) for a canonical d-connection D

$\mathbf{R}_{\alpha \beta}=\Upsilon_{\alpha \beta}$.

Such equations are equivalent to (6) if there are imposed additional nonholonomic constraints, or found some smooth limits, for extracting LC-configurations, $\mathbf{D}_{\mid \widehat{\mathcal{T}}=0}=\nabla$, for instance, of type

$\mathbf{T}_{\alpha \beta}^{\gamma}=0$.

In (7), a matter fields source $\boldsymbol{\Upsilon}_{\mu \nu}$ can be constructed using a N-adapted variational calculus for ${ }^{m} \mathcal{L}\left(\mathbf{g}, \widehat{\mathbf{D}},{ }^{A} \varphi\right)$, when $\boldsymbol{\Upsilon}_{\mu \nu}=\varkappa\left({ }^{m} \mathbf{T}_{\mu \nu}-\frac{1}{2} \mathbf{g}_{\mu \nu}{ }^{m} \mathbf{T}\right) \rightarrow \varkappa\left({ }^{m} T_{\mu \nu}-\frac{1}{2} \mathbf{g}_{\mu \nu}{ }^{m} T\right)$ for [coefficients of $\mathbf{D}] \rightarrow$ [coefficients of $\nabla$ ] even, in general, $\mathbf{D} \neq \nabla$. In such formulas, we consider ${ }^{m} \mathbf{T}=\mathbf{g}^{\mu \nu m} \mathbf{T}_{\mu \nu}$ for

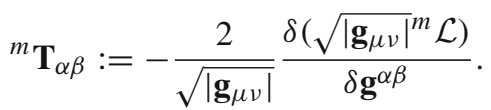

We note that any (pseudo) Riemannian geometry and gravity theory, and various metric-affine modifications (for instance, $F(R)$-modified theories $[63,68]$ ), can be formulated equivalently using geometric data $(\mathbf{g}, \nabla)$ and/or $(\mathbf{g}, \mathbf{D})$. There is an important motivation to use nonholonomic variables of type $(\mathbf{g}, \mathbf{D})$ because that they allow to decouple and integrate in general form various modified and standard Einstein equation. Such solutions can be with generic offdiagonal metrics and coefficients depending on all spacetime coordinates [55-57]. A recent review of the so-called anholonomic frame deformation method, AFDM, of constructing

\footnotetext{
7 We use abstract left labels $A$ and $m$ in order to distinguish the values from similar notations of pure geometric objects, for instance, $\mathbf{T}_{\alpha \beta}^{\gamma}$.
} 
exact solutions in GR and MGTs, geometric flow theory, and applications in modern cosmology and astrophysics can be found in [63]. In this work, we shall develop the AFDM for constructing exact solutions in entropic geometric flow and gravity theories.

\subsection{Nonholonomic $3+1$ splitting adapted to $2+2$ structures}

We outline some basic concepts on the geometry of $3+1$ foliations of a nonholonomic Lorentzian manifold $(\mathbf{V}, \mathbf{g}, \mathbf{N})$ of signature $(+++-)$ into a family of non-intersecting space like 3-d hypersurfaces $\Xi_{t}$ parameterized by a "time function", $t\left(u^{\alpha}\right)$, stated as a scalar field as described as follows. Such spacetime decompositions are useful for elaborating various thermodynamic, locally anisotropic kinetic [71] and geometric evolution or hydrodynamic flow models [38] when a conventional splitting into time and space like coordinates is necessary. This allows definition of physical important values (for instance, entropy, effective energy etc.) and deriving fundamental geometric evolution equations. In our approach, we generalize the well-known geometric $3+1$ formalism in GR (see, for instance, [72]) to the case of nonholonomic manifolds [38-40].

For a 3-d manifold , $\Xi$, we consider an one-to-one image to a hypersurface $\Xi=\zeta(, \Xi) \subset \mathbf{V}$ constructed as an homeomorphism with both continuous maps $\zeta$ and $\zeta^{-1}$, when $\Xi$ does not intersect itself. Left "up" or "low" labels by a vertical bar ", " will be used in order to emphasize that certain geometric objects refer to 3-d manifolds/hypersurfaces. Such a 3-d space is supposed to be locally defined as a set of points for which a scalar field $t$ on $\mathbf{V}$ is constant (for instance, i.e. $t(p)=0$ for any point $p \in \Xi)$. It is assumed also that $t$ spans the real line $\mathbb{R}$ and that any $\Xi$ is a connected submanifold of $\mathbf{V}$ with the topology of $\mathbb{R}^{3}$.

It should be noted that any $2+2$ splitting by a nonholonomic distribution $\mathbf{N}$ (1) induces a N-connection structure for a hypersurface $\Xi$, i.e. an induced $\mathrm{N}$-connections ${ }_{\text {}} \mathbf{N}: T, \Xi=h, \Xi \oplus v, \Xi$. Using the coefficients of such an induced $\mathrm{N}$-connection, any induced 3-metric tensor $\mathbf{q}$ can be

\footnotetext{
${ }^{8}$ We can label local coordinates for a $3+1$ splitting in $u^{\alpha}=\left(x^{\grave{\imath}}, t\right)$, where indices $\alpha, \beta, \ldots=1,2,3,4$ and $\grave{i}, \grave{j}, \ldots=1,2,3$ are related to a $2+2$ splitting as in previous subsection (in brief, we shall write $u=(\breve{u}, t))$. The continuous maps $\zeta$ can be parameterized to "carry along" curves/vectors in , $\Xi$ to curves/vectors in $\mathbf{V}$, for $\zeta:\left(x^{\imath}\right) \longrightarrow$ $\left(x^{i}, 0\right)$. This way, it is possible to define and relate respective local bases $\partial_{\grave{l}}:=\partial / \partial x^{\grave{\imath}} \in T(, \boldsymbol{\Xi})$ and $\partial_{\alpha}:=\partial / \partial u^{\alpha} \in T \mathbf{V}$. The coefficients of 3 -vectors and 4 -vectors are expressed correspondingly,, $\mathbf{a}=a^{i} \partial_{i}$ and $\mathbf{a}=a^{\alpha} \partial_{\alpha}$ (we shall use also capital letters, for instance,, $\mathbf{A}=A^{i} \partial_{i}$ and $\mathbf{A}=A^{\alpha} \partial_{\alpha}$ ). Similar formulas are considered for dual forms to vectors, 1 -forms, when the dual bases $\mathbf{d} x^{i} \in T^{*}(, \boldsymbol{\Xi})$ and $d u^{\alpha} \in T^{*} \mathbf{V}$. The 1-forms will be parameterized for respective 3 and 4 dimensions, ${ }_{1} \tilde{\mathbf{A}}=A_{i} \mathbf{d} x^{i}$ and $\tilde{\mathbf{A}}=A_{\alpha} d u^{\alpha}$. We shall omit the left/up label by a tilde $\sim$ (writing, $\mathbf{A}$ and $\mathbf{A})$ if that will not result in ambiguities.
}

written in $\mathrm{N}$-adapted frames as a d-tensor (d-metric) in the form

$$
\begin{aligned}
\mathbf{q}= & (h \mathbf{q}, v \mathbf{q})=q_{i}(u) \mathbf{e}^{\grave{i}} \otimes \mathbf{e}^{i}=q_{i}\left(x^{k}\right) d x^{i} \otimes d x^{i} \\
& +q_{3}\left(x^{k}, y^{3}\right)_{1} \mathbf{e}^{3} \otimes{ }_{1} \mathbf{e}^{3}, \\
& \text { for }{ }_{1} \mathbf{e}^{3}=d u^{3}+{ }_{1} N_{i}^{3}(u) d x^{i},
\end{aligned}
$$

where ${ }_{1} N_{i}^{3}(u)$ can be identified with $N_{i}^{3}(u)$ choosing common frame and coordinate systems for $\Xi \subset \mathbf{V}$. We can extend naturally such a 3-d metric $\mathbf{q}$ to a 4-d d-metric $\mathbf{g}$ (2) reparameterized in a form adapted both to $2+2$ and $3+1$ nonholonomic splitting,

$$
\begin{aligned}
\mathbf{g} & =(h \mathbf{g}, v \mathbf{g})=\breve{g}_{\grave{i} j} \mathbf{e}^{\grave{\imath}} \otimes \mathbf{e}^{\grave{j}}+g_{4} \mathbf{e}^{4} \otimes \mathbf{e}^{4} \\
& =q_{i}(u) \mathbf{e}^{\grave{i}} \otimes \mathbf{e}^{\grave{i}}-\breve{N}^{2} \mathbf{e}^{4} \otimes \mathbf{e}^{4}, \\
\mathbf{e}^{3} & ={ }_{\imath} \mathbf{e}^{3}=d u^{3}+{ }_{1} N_{i}^{3}(u) d x^{i}, \\
\mathbf{e}^{4} & =\delta t=d t+N_{i}^{4}(u) d x^{i} .
\end{aligned}
$$

For $\mathbf{g}(10)$, the lapse function $\breve{N}(u)>0$ is defined as a positive scalar field which ensues that the d-vector $\mathbf{n}$ is a unite one. An "inverse hat" symbol is used in order to distinguish such a symbol from $N$ is used traditionally in literature on GR [72]. Here we note that in another turn, the symbol $N_{i}^{a}$ is used traditionally for the $\mathrm{N}$-connection and this also motivates a new symbol $\breve{N}$.

We note that for any quadratic line element $d s^{2}=$ $g_{\alpha \beta} d u^{\alpha} d u^{\beta}$ of a metric tensor $\mathbf{g}$ there are such frame transforms to parameterizations when $\breve{g}_{i j}=q_{\grave{i}}=g_{\alpha \beta} e_{i}^{\alpha} e_{j}^{\beta}$ is just the induced metric on $\Xi_{t}$. In result, the determinants of 4-d and 3-d metrics are computed $\sqrt{|g|}=\breve{N} \sqrt{|\breve{g}|}=\breve{N} \sqrt{|q|}$. Using certain coordinates $\left(x^{\imath}, t\right)$ being $\mathrm{N}$-adapted on respective hypersurfaces, the time partial derivatives are computed $£_{t} q=\partial_{t} q=q^{*}$ and the spacial derivatives are computed $q_{, i}:=e_{i}^{\alpha} q_{, \alpha}$.

There are two types of induced linear connections completely determined by an induced 3-d hypersurface metric $\mathbf{q}$,

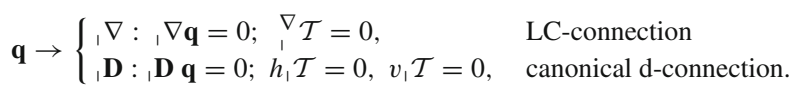

Such formulas are related to 4-d similar ones (4). Both linear connections, , $\nabla$ and, $\mathbf{D}$, are subjected also to a distortion relation ${ }_{1} \mathbf{D}\left[\mathbf{q},{ }_{1} \mathbf{N}\right]={ }_{1} \nabla[\mathbf{q}]+{ }_{1} \mathbf{Z}\left[{ }_{1} \mathcal{T}\left(q,{ }_{1} \mathbf{N}\right)\right]$.

For 3-d configurations, we can compute the $\mathrm{N}$-adapted coefficient formulas for nonholonomically induced torsion structure ${ }_{1} \mathbf{T}=\left\{{ }_{1} \mathbf{T}_{j \grave{k}}^{\grave{ }}{ }_{j k}\right.$, determined by ${ }_{1} \mathbf{D}$, and for the Riemannian tensors $\left.{ }_{1} R={ }_{1} R^{i}{ }_{j k k}\right\}$ and $\left.{ }_{1} \mathbf{R}={ }_{1} \mathbf{R}^{i}{ }_{j k k}\right\}$, determined respectively by,$\nabla$ and ,D. Using 3 -d subsects of coefficient formulas, we can compute respective $\mathrm{N}$-adapted

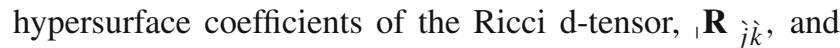
the Einstein d-tensor, ${ }_{1} \mathbf{E}_{\dddot{j} k}$. Contracting indices, we obtain 
the Gaussian curvature, $R=q^{\grave{j}{ }_{1}} R_{\grave{j} k}$, and the Gaussian canonical curvature, ${ }_{1}^{s} R=q^{\grave{j}{ }_{1}} \mathbf{R}_{j \grave{k}}$, of $\left(\Xi, \mathbf{q},{ }_{1} \mathbf{N}\right)$. It should be noted that all this types of $\mathrm{N}$-adapted and not $\mathrm{N}$-adapted geometric objects can be defined in abstract form which do not depend on the type of embedding of a nonholonomic 3-d manifold $\left(\boldsymbol{\Xi}, \mathbf{q},{ }_{1} \mathbf{N}\right)$ into a 4 -d one $(\mathbf{V}, \mathbf{g}, \mathbf{N})$.

\subsection{Quasiperiodic space and time QC configurations}

Let us consider two examples of space and time quasiperiodic structures defined in a curved spacetime following our works on quasicrystal, QC, models in modern cosmology $[61,63,64]$ (alternative models are studied in [73]). Our approach was elaborated following Wilczek and co-authors works in condensed matter physics [74-77]. As a toy model, we consider one dimensional, 1-d, time quasicrystals, TQCs with time structure equations generalizing those introduced in [77]). Then we introduce some important formulas on three dimensional, 3-d, QC structures - in general, such configurations are called space-time quasicrystal structures, STQC, and studied in [64]. In this work we use a different system of notation for partial derivatives when, for instance, $\partial q / \partial x^{i}=$ $\partial_{i} q, \partial q / \partial y^{3}=\partial_{3} q=q^{*}$, and $\partial q / \partial y^{4}=\partial_{4} q=\partial_{t} q=q^{\star}$, for a function $q\left(x^{i}, y^{3}, t\right)$.

\subsubsection{1-d relativistic time QC structures}

We consider a scalar field $\varsigma\left(x^{i}, y^{a}\right)$ on a space-time $(\mathbf{V}, \mathbf{g}, \mathbf{N})$ and respective Lagrange density

$$
\begin{aligned}
\dot{L}(\varsigma)= & \frac{1}{48}\left(\mathbf{g}^{\alpha \beta}\left(\mathbf{e}_{\alpha} \varsigma\right)\left(\mathbf{e}_{\beta} \varsigma\right)\right)^{2} \\
& -\frac{1}{4} \mathbf{g}^{\alpha \beta}\left(\mathbf{e}_{\alpha} \varsigma\right)\left(\mathbf{e}_{\beta} \varsigma\right)-\dot{V}(\varsigma) .
\end{aligned}
$$

In this formula, $\dot{V}(\varsigma)$ is a nonlinear potential and $\mathbf{e}_{\alpha}$ are $\mathrm{N}$ adapted partial derivatives. Corresponding $\mathrm{N}$-adapted variational motion equations are $\left[\frac{1}{2} \mathbf{g}^{\alpha \beta}\left(\mathbf{e}_{\alpha} \varsigma\right)\left(\mathbf{e}_{\beta} \varsigma\right)-1\right]\left(\mathbf{D}^{\gamma} \mathbf{D}_{\gamma} \varsigma\right)$ $=2 \frac{\partial \dot{V}}{\partial \varsigma}$. The field $\varsigma$ defines a 1-d time QC structure, 1-TQC, if it is a solution of these motion equations. ${ }^{9}$

\subsubsection{3-d QC structures on curved spaces}

QC structures and analogous dynamic phase field crystal models can be elaborated as flow evolution theories on real parameter $\tau$ (in next section, this parameter will be identified with a geometric flows one). Such a QC structure can be

\footnotetext{
${ }^{9}$ For non-relativistic limits with $g_{\alpha \beta}=[1,1,1,-1]$ and $\varsigma \rightarrow \varsigma(t)$, $\dot{L} \rightarrow \frac{1}{12}\left(\varsigma^{\bullet}\right)^{4}-\frac{1}{2}\left(\varsigma^{\bullet}\right)^{2}-\dot{V}(\varsigma)$, which leads to an effective energy $E=$ $\frac{1}{4}\left[\left(\varsigma^{\bullet}\right)^{2}-1\right]^{2}+\dot{V}(\varsigma)-\frac{1}{4}$ and motion equations $\left[\left(\varsigma^{\bullet}\right)^{2}-1\right] \varsigma^{\bullet \bullet}=-\frac{\partial \dot{V}}{\partial \zeta}$ introduced in [77]. The Lagrange density (12) provides a generalization for 1-TQCs modeled on a curved spacetime which can be also modeled in entropic gravity theories.
}

defined by a generating function $\bar{q}=\bar{q}\left(x^{i}, y^{3}, \tau\right)$ subjected to the condition that it is a solution of an evolution equation with conserved dynamics,

$\frac{\partial \bar{b}}{\partial \tau}={ }_{,} \widehat{\Delta}\left[\frac{\delta \bar{F}}{\delta \bar{b}}\right]=-{ }_{,} \widehat{\Delta}\left(\Theta \bar{b}+Q \bar{b}^{2}-\bar{b}^{3}\right)$.

Such evolution is considered on 3-d spacelike hypersurface $\Xi_{t}$ when the canonically nonholonomically deformed hypersurface Laplace operator $\widehat{ }_{1}:=\left({ }_{1} \mathbf{D}\right)^{2}=q^{i \grave{j}}{ }_{1} \mathbf{D}_{i}, \mathbf{D}_{\grave{j}}$, where indices rung values $i, \grave{j}, \ldots 1,2,3$. This operator is a distortion of $, \Delta:=(, \nabla)^{2}$ constructed in 3-d Riemannian geometry, see previous subsection. The functional $\bar{F}$ in (13) is characterized by an effective free energy

$\bar{F}[\bar{q}]=\int\left[-\frac{1}{2} \bar{b} \Theta \bar{b}-\frac{Q}{3} \bar{b}^{3}+\frac{1}{4} \bar{b}^{4}\right] \sqrt{q} d x^{1} d x^{2} \delta y^{3}$,

where $q=\operatorname{det}\left|q_{i}\right|, \delta y^{3}=\mathbf{e}^{3}$ and the operators $\Theta$ and $Q$ are defined and explained in $[63,64]$. Such nonlinear interactions are stabilized by the cubic term with $Q$ and the second order resonant interactions are varied by setting observable values of such constants (they are different for cosmological models, in astrophysics or condensed matter physics). The average value $\langle\bar{b}\rangle$ is conserved for any fixed time variable $t$ and/or evolution parameter $\tau_{0}$. We can fix $\langle\bar{b}\rangle_{\mid \tau=\tau_{0}}=0$ when other values are accommodated by redefining values $\Theta$ and $Q$.

\subsection{Distributions defining spacetime elastic configurations}

In letter [32], we shown that models of entropic gravity can be derived from nonholonomic modifications of the $\mathrm{W}$ functional when the (modified) Einstein equations are equivalent to certain nonholonomic Ricci soliton equations. Here, we shall study the conditions when entropic elastic scenarios can be modelled as nonholonomic Ricci solitons in Sect. 3.3. We shall consider certain examples of nonholonomic distributions and related Lagrange densities on a Lorentz manifold $\mathbf{V}$ which are used in entropic gravity theories $[6,7,32,65]$. Using such geometric constructions, we shall elaborate in next section on elastic flow evolution models and their selfsimilar nonholonomic Ricci soliton configurations. There are three important values:

$\varepsilon_{\alpha \beta}=\mathbf{D}_{\alpha} \mathbf{u}_{\beta}-\mathbf{D}_{\beta} \mathbf{u}_{\alpha}$ - the elastic strain tensor;

$\phi=u / \sqrt{|\Lambda|}$ - a dimensionless scalar;

$\chi=\alpha\left(\mathbf{D}_{\mu} \mathbf{u}^{\mu}\right)\left(\mathbf{D}_{v} \mathbf{u}^{v}\right)+\beta\left(\mathbf{D}_{\mu} \mathbf{u}_{v}\right)\left(\mathbf{D}^{\mu} \mathbf{u}^{v}\right)$

$+\gamma\left(\mathbf{D}_{\mu} \mathbf{u}_{\nu}\right)\left(\mathbf{D}^{\nu} \mathbf{u}^{\mu}\right)$ - a general kinetic term for $\mathbf{u}^{\mu}$.

These geometric/physical objects are determined by a conventional displacement vector field $\mathbf{u}^{\alpha}$, cosmological constant $\Lambda$ and some constants $\alpha, \beta, \gamma$; there are used short hand notations: $u:=\sqrt{\left|\mathbf{u}_{\alpha} \mathbf{u}^{\alpha}\right|}, \varepsilon=\varepsilon_{\beta}^{\beta}$, and $\mathbf{n}^{\alpha}:=\mathbf{u}^{\alpha} / u$. 
On $\mathbf{V}$, there are considered nonholonomic distributions for corresponding total, effective gravitational, usual matter, interaction and kinetic terms of Lagrangians postulated in the form

$$
\begin{aligned}
& { }^{\text {tot }} \mathcal{L}={ }^{g} \mathcal{L}+{ }^{m} \mathcal{L}+{ }^{i n t} \mathcal{L}+{ }^{\chi} \mathcal{L} \\
& \text { for }{ }^{g} \mathcal{L}=M_{P}^{2} F\left({ }^{s} R\right), \quad{ }^{i n t} \mathcal{L}=-\sqrt{|\Lambda|}{ }^{m} \mathbf{T}_{\mu \nu} \mathbf{u}^{\mu} \mathbf{u}^{v} / u \\
& { }^{\chi} \mathcal{L}=M_{P}^{2}|\Lambda|\left(\chi^{3 / 2}+|\Lambda||u[\varsigma, \bar{b}]|^{2 z}\right)
\end{aligned}
$$

In these formulas, the Plank gravitational mass is denoted $M_{P}$ and the gravitational Lagrangian ${ }^{g} \mathcal{L}$ is taken as in modified gravity $[63,68-70]$. We can fix $z=1$ if we search for compatibility with [65], or $z=2$ if we search for a limit to the standard de Sitter space solution [66,67] (as we use in [32]). To model STQC structures in entropic gravity and related geometric flow theories we can consider that the displacement vector field $\mathbf{u}^{\alpha}[\varsigma, \bar{b}]$ is a functional of functions $\varsigma, \bar{b}$ subjected to certain conditions of type (12) and/or (13) [in principle, we can consider functionals for pattern forming, nonlinear wave soliton structures, fractional and diffusion processes etc.].

The energy-momentum tensors considered in above formulas and/or derived from respective Lagrangians in (14) and computed using variations on $\mathbf{g}^{\mu \nu}$ similarly to ${ }^{m} \mathbf{T}_{\mu \nu}$ (9) (in $\mathrm{N}$-adapted form, details of such computations are provided in $[39,58,59,63])$. For the full system, the effective energy-momentum tensor is computed

$$
\begin{gathered}
{ }^{t o t} \mathbf{T}_{\mu \nu}=\left(\frac{\partial F}{\partial^{s} R}\right)^{-1}{ }^{m} \mathbf{T}_{\mu \nu}+{ }^{F} \mathbf{T}_{\mu \nu}+{ }^{i n t} \mathbf{T}_{\mu \nu}+{ }^{\chi} \mathbf{T}_{\mu \nu} \\
\text { where }{ }^{F} \mathbf{T}_{\beta \gamma}=\left[\frac{1}{2}\left(F-\frac{\partial F}{\partial^{s} R}\right) \mathbf{g}_{\beta \gamma}\right. \\
\left.-\left(\mathbf{g}_{\beta \gamma} \mathbf{D}_{\alpha} \mathbf{D}^{\alpha}-\mathbf{D}_{\beta} \mathbf{D}_{\gamma}\right) \frac{\partial F}{\partial^{s} R}\right]\left(\frac{\partial F}{\partial^{s} R}\right)^{-1}
\end{gathered}
$$

We can model "pure" elastic spacetime modifications of the Einstein gravity if we fix $F\left({ }^{s} R\right)={ }^{s} R$ and consider restrictions to the Levi-Civita connection $\mathbf{D}=\nabla$. For such conditions, we obtain respective formulas for ${ }^{i n t} \mathbf{T}_{\mu \nu}$ and $\chi \mathbf{T}_{\mu \nu}$ which are similar to formulas (10)-(13) in [66]. ${ }^{10}$

In this work, the generalized (effective) source for MGT (7) splits into four components,

$$
\begin{aligned}
{ }^{t o t} \boldsymbol{\Upsilon}_{\mu \nu}:= & \varkappa\left({ }^{t o t} \mathbf{T}_{\mu \nu}-\frac{1}{2} \mathbf{g}_{\mu \nu}{ }^{t o t} \mathbf{T}\right) \\
= & \left(\frac{\partial F}{\partial^{s} R}\right)^{-1}{ }^{m} \boldsymbol{\Upsilon}_{\mu \nu}+{ }^{F} \boldsymbol{\Upsilon}_{\mu \nu} \\
& +{ }^{i n t} \boldsymbol{\Upsilon}_{\mu \nu}+{ }^{\chi} \boldsymbol{\Upsilon}_{\mu \nu},
\end{aligned}
$$

where $\varkappa$ is determined in standard form by the Newton gravitational constant $G$. We need additional terms and parame-

\footnotetext{
$\overline{10}$ We use a system of notations which is similar (but without "hats") to [35, 38-40,63]; such notations are different from those used in [65-67].
}

terizations in order to describe structure formation in modern cosmology and to model dark energy and dark matter properties.

\section{Relativistic geometric flows and modified entropic gravity}

Grigory Perelman's proof of the Poincaré conjecture [45] on geometric flow evolution of 3-d Riemannian metrics [4244] provided fundamental results in geometric analysis and topology. There were also studied possible applications in modern mathematical and particle physics. We cite [46-48] for reviews of rigorous mathematical results. For early applications, we refer to D. Friedan works [49-51] (he considered geometric evolution equations related to renorm group equations before the Hamilton-Poincaré theory was elaborated). Further developments and applications were performed in [52-54] and a series of works [33-41], see also references therein. In those works on theories of nonholonomic/noncommutative/supersymmetrics, fractional, diffusion etc. geometric flows, there were studied statistical and thermodynamic evolution models derived from certain Lyapunov type functionals. Such F- and W-entropy functionals are called in literature the Perelman functionals. The Wentropy has properties of "minus entropy" of statistical thermodynamics systems. In [32], we elaborate on the idea that such a W-entropy can be considered for formulating Verlinde type entropic gravity theories [6,7]. We study self-similar configurations of nonholonomic geometric flows resulting in entropic Ricci solitons (see Sect. 3.3).

We note that Perelman suggested in his first preprint [45] that the geometric flow theory may have certain implications in black hole physics and string theory. Nevertheless, the original theory of Ricci flows was formulated in a nonrelativistic form. To consider further generalizations and applications in modern physics and cosmology we elaborated on relativistic models of geometric flow theories [3840]. Such constructions can be re-defined for nonholonomic configurations modeling elastic and quasiperiodic spacetime structure as in Sect. 2.4 and allows rigorous geometric motivations for emergent entropic theories of type $[6,7,32,65]$.

The goal of this section is to study generalizations of the hypersurface 3-d and relativistic 4-d F- and W-functionals and elaborate on respective geometric evolution scenarios supporting the Verlinde entropic gravity conjecture [6,7]. Such constructions can be considered in the framework of $s$ a modified relativistic variant of the Poincaré-Thurston conjecture which was proven only for certain classes of Riemannian and Kähler metrics, see details in [45-48]. For relativistic configurations, we can only elaborate on geometric evolution of certain 3-d hypersurface configurations subjected to the conditions that such 3 -metrics can be extended to cer- 
tain classes of 4-d metric and (non) linear connection structures derived as exact/parametric solutions of certain nonholonomic/entropic geometric flow equations. There will be considered also generalizations of the Hamilton equations for the entropic flow theory. The conditions for generating entropic modified Einstein equations as nonholonomic Ricci solitons will be also analysed. We emphasize that in the main part of this article there are studied relativistic geometric flow models with a temperature like evolution parameter.

\subsection{Modified spacetime and hypersurface Perelman's functionals}

Let us consider families of nonholonomic 4-d manifolds $\mathbf{V}(\tau)$ parameterized by a positive parameter $\tau, 0 \leq \tau \leq \tau_{0}$ (it can be considered as a temperature like parameter) and enabled with a double nonholonomic $2+2$ and $3+1$ splitting [38-40]. Such manifolds are determined by respective families of metrics $\mathbf{g}(\tau)=\mathbf{g}(\tau, u)$ and N-connections $\mathbf{N}(\tau)=\mathbf{N}(\tau, u)$ (we shall write only the parametric dependence if that will not result in ambiguities) for which canonically corresponding d-connection structures can be constructed $\mathbf{D}(\tau)=\mathbf{D}(\tau, u)$. We also suppose that on $\mathbf{V}(\tau)$ there are defined corresponding families of Lagrange densities ${ }^{g} \mathcal{L}(\tau)$, for gravitational fields in a MGT or GR, and ${ }^{\text {tot }} \mathcal{L}(\tau)$, as total Lagrangians for effective and matter fields (14). For a double $2+2$ and $3+1$ splitting, we can consider local coordinates labeled as $u^{\alpha}=\left(x^{i}, y^{a}\right)=\left(x^{\grave{\imath}}, u^{4}=t\right)$ for $i, j, k, \ldots=1,2 ; a, b, c, \ldots=3,4$; and $\grave{i}, \grave{j}, \grave{k}=1,2,3$. The nonholonomic distributions for $\mathrm{N}$-connections can be parameterized always in such forms that any open region $U \subset \mathbf{V}$ is covered by a family of 3-d spacelike hypersurfaces $\Xi_{t}$ parameterized by a time like parameter $t$.

\subsubsection{Generalized Perelman functionals for entropic geometric flows and MGTS}

For this class of theories, we postulate the modified Perelman's functionals in the form

$$
\begin{aligned}
\mathcal{F}(\tau)= & \int_{t_{1}}^{t_{2}} \int_{\Xi_{t}} e^{-f} \sqrt{|\mathbf{g}|} d^{4} u \\
& \times\left[F\left({ }^{s} R\right)+{ }^{t o t} \mathcal{L}+|\mathbf{D} f|^{2}\right] \text { and } \\
\mathcal{W}(\tau)= & \int_{t_{1}}^{t_{2}} \int_{\Xi_{t}}(4 \pi \tau)^{-3} e^{-f} \sqrt{|\mathbf{g}|} d^{4} u \\
& \times\left[\tau\left(F\left({ }^{s} R\right)+{ }^{t o t} \mathcal{L}+|h \mathbf{D} f|+|v \mathbf{D} f|\right)^{2}+f-8\right] .
\end{aligned}
$$

The condition $\int_{t_{1}}^{t_{2}} \int_{\Xi_{t}}(4 \pi \tau)^{-3} e^{-f} \sqrt{|\mathbf{g}|} d^{4} u=1$ is imposed on the normalizing function $f(\tau, u)$. For topological considerations, such a normalisation is not important. Nevertheless, it imposes certain nonholonomic constraints on geo- metric objects which do not allow to solve derived geometric flow evolution equations in explicit form. For applications to entropic gravity and associated thermodynamic models, we can consider $f$ as an undetermined scalar function which can be related to possible conformal transforms or re-parameterizations. In result, we can prove certain general decoupling and integration properties of corresponding systems of nonlinear PDEs. Fixing a class of solutions, we can chose such integration functions and constants which reproduce/predict certain experimental and/or observational data. Corresponding values of $f$ depend on systems of reference and coordinates.

Let us explain and motivate the difference of (16) and (17), introduced in the first partner work [32], from the original Grisha Perelman F- and W-functionals [45] postulated for the Ricci flows of 3-d Riemannian metrics, see details in monographs [46-48]. In this work, we study geometric entropic flows of canonical geometric data $(\mathbf{g}(\tau), \mathbf{N}(\tau), \mathbf{D}(\tau))$ for nonholonomic Lorentz manifolds and various generalizations for MGTs following the program elaborated in [35,3840,63 ], where possible connections to emergent gravity were not analyzed. In formulas (16) and (17), we consider the gravitational Lagrangian ${ }^{g} \mathcal{L}=F\left({ }^{s} R\right)$ as a functional of the scalar curvature for $\mathbf{D}$, or ${ }^{g} \mathcal{L}=R[\nabla]$ for considering as particular cases models of geometric evolution of exact solutions in GR. The key difference from previous works is that in such relativistic functionals the term ${ }^{\text {tot }} \mathcal{L}$ is introduced, which is responsible for geometric evolution of configurations with elasticity and quasiperiodicity. Those functionals can be generalized on a temperature like parameter $\tau$ and used as certain alternative geometric functionals, for instance, for W-entropy. Nevertheless, only nonholonomic elastic quasiperiodic functionals of type (16) and (17) result for self-similar configurations (see next subsections) in entropic gravity equations of Verlinde type [6,7,32,65] and/or with quasiperiodic structure $[61,63,64]$.

In this and partner [32] papers, we work with generalized geometric flow and entropy functionals determined by $F\left({ }^{s} R\right)+{ }^{\text {tot }} \mathcal{L}$ and $\mathbf{D}$, respectively, instead of the Riemannian values $R$ and $\nabla$ used in the former mathematical works. In our nonholonomic approaches, above F- and W-functionals characterize relativistic thermodynamic models with analogous nonlinear hydrodynamic flows of families of entropic values, metrics and generalized connections, encoding interactions of gravitational and matter fields as it is motivated in [38-40]. In general, it is possible to work with any class of normalizing functions $f(\tau, u)$ which can be redefined in order to include geometric and matter Lagrange terms and certain constant values and parameters. In many cases, such a function is chosen in a non-explicit form. This allows us to study non-normalized geometric flows but with nonholonomic constraints. For such conditions, there found various general decoupling and integration properties of respective 
physically important systems of nonlinear PDEs. In result, generic off-diagonal solutions can be constructed in explicit form as in [32,35,38-40,63], but with entropic and quasiperiodic modifications. The existence of such solutions validates our nonholonomic geometric flow entropic approach, involving metrics with pseudo-Euclidean signature even analogs of the Poincaré-Thurston conjecture have not been formulated and proven for the Lorentzian spacetimes. Nevertheless, explicit constructions of exact solutions with elastic and quasiperiodic gravitational metrics and effective matter sources, which will be provided in Sects. 4-6, support E. Velinde conjecture on entropic gravity which in our works is proven for modified Poincaré functionals.

\subsubsection{Nonholonomic 3-d space like hypersurface F-and W-functionals}

We can redefine and compute relativistic entropies (16) and (17) for any $3+1$ splitting with 3 -d closed hypersurface fibrations $\widehat{\Xi}_{t}$ as we described above in Sect. 2.2.

Let us denote by ${ }_{1} \mathbf{D}=\mathbf{D}_{\mid \widehat{\Xi}_{t}}$ the canonical d-connection D defined on a 3-d hypersurface $\widehat{\Xi}_{t}$, when all values depend on a temperature like parameter $\tau\left(\tau^{\prime}\right)$ with possible scale re-definitions for another parameter $\tau^{\prime}$ etc. We define also ${ }_{1}^{s} R:={ }^{s} R_{\mid \widehat{\Xi}_{t}}$. Using $q_{i}(\tau)=\left[q_{i}(\tau), q_{3}(\tau)\right]$ in a family of d-metrics (10), the Perelman's functionals parameterized in $\mathrm{N}$-adapted form are constructed in the form:

$$
\begin{aligned}
, \mathcal{F}= & \int_{\widehat{\Xi}_{t}} e^{-, f} \sqrt{\left|q_{i j}\right|} d \grave{x}^{3} \\
& \times\left[\left({ }_{1} F\left({ }_{1}^{s} R\right)+{ }_{1}^{t o t} \mathcal{L}+\left|, \mathbf{D}_{l} f\right|^{2}\right)\right], \text { and } \\
\mathcal{W}= & \int_{\widehat{\Xi}_{t}} \mu \sqrt{\left|q_{i j}\right|} d \grave{x}^{3} \\
& \times\left[\tau\left(\left({ }_{1} F\left({ }_{1}^{s} R\right)+{ }_{1}^{\text {tot }} \mathcal{L}+\left|{ }_{1}^{h} \mathbf{D} f\right|+\left|{ }_{1}^{v} \mathbf{D}_{1} f\right|\right)^{2}+{ }_{1} f-6\right] .\right.
\end{aligned}
$$

These functionals are derived respectively from the previous 4-d elastic ones when the values, $F\left({ }_{1}^{s} R\right)$ and ${ }_{1}^{\text {tot }} \mathcal{L}$ are computed as projections on a 3-d hypersurface for a redefined normalization function, $f$. Using frame/coordinate transform and re-definition of the temperature like parameter, we can always chose a necessary type scaling function , $f$ which satisfies normalization conditions $\int_{\widehat{\Xi}_{t},} \mu \sqrt{\left|q_{i j}\right|} d \grave{x}^{3}=1$ for ${ }_{\text {, }} \mu=(4 \pi \tau)^{-3} e^{-, f}$.

The functionals (18) and (19) transform into standard Perelman functionals [45] for 3-d Riemannian metrics on $\widehat{\Xi}_{t}$ if ${ }_{1} \mathbf{D} \rightarrow, \nabla,{ }_{1} F\left({ }_{1}^{s} R\right)={ }_{1}^{s} R$ and ${ }_{1}^{\text {tot }} \mathcal{L}=0$. In order to describe possible contributions on 3-d hypersurfaces of spacetime elasticity and quasiperiodic structure in entropic gravity, it is necessary to analyze physical effects of such nonholonomic deformations.
3.2 Geometric flow equations for modified gravitational and matter fields

Applying a variational procedure for a corresponding Ffunctional for geometric flows of 3-d Riemannian metrics, Perelman [45] provided a proof for R. Hamilton's equations [42-44]. For self-consistent configurations with a fixed flow parameter $\tau_{0}$, one obtains 3-d Ricci soliton equations which are equivalent to the vacuum Einstein equations for $\nabla$ with an effective cosmological constant. In similar forms to rigorous mathematical proofs in [45-48] but elaborating on $\mathrm{N}$-adapted variational procedures, for instance, for the functional $\mathcal{F}(\tau)$ (16) with a canonical $\mathbf{D}$ used instead of $\nabla$ (see details in $[32,38-40]$ ), we obtain a system of nonlinear PDEs generalizing the R. Hamilton equations for entropic and quasiperiodic geometric flow evolution determined by canonical data $\left(\mathbf{g}=\left\{\mathbf{g}_{\mu \nu}=\left[g_{i j}, g_{a b}\right]\right\}, \mathbf{N}=\left\{N_{i}^{a}\right\}, \mathbf{D},{ }^{t o t} \mathcal{L}\right)$,

$$
\begin{aligned}
\partial_{\tau} g_{i j} & =-2\left(\mathbf{R}_{i j}-{ }^{t o t} \Upsilon_{i j}\right) \\
\partial_{\tau} g_{a b} & =-2\left(\mathbf{R}_{a b}-{ }^{t o t} \Upsilon_{a b}\right) ; \\
\mathbf{R}_{i a} & =\mathbf{R}_{a i}=0 ; \quad \mathbf{R}_{i j}=\mathbf{R}_{j i} ; \quad \mathbf{R}_{a b}=\mathbf{R}_{b a} ; \\
\partial_{\tau} f & \left.=-\widehat{\square} f+|\mathbf{D} f|^{2}-{ }^{s} R+{ }^{t o t} \Upsilon_{\alpha}^{\alpha}\right)
\end{aligned}
$$

In these formulas, $\widehat{\square}(\tau)=\mathbf{D}^{\alpha}(\tau) \mathbf{D}_{\alpha}(\tau)$ and ${ }^{t o t} \Upsilon_{\alpha \beta}(\tau)$ is chosen for geometric flows of (effective) sources of entropic gravity (15) (if we fix any $\tau=\tau_{0}$ ). We note that the dependence on a flow parameter $\tau$ for such (effective) matter sources is determined by certain evolutions of $\mathbf{g}(\tau)$ and $\mathbf{D}(\tau)$. In such theories, we do not consider nonholonomic deformations and evolution of classical matter fields. For instance, we do not consider geometric flow evolution equations for the electromagnetic potential $\mathbf{A}_{\alpha}(\tau)$ with evolution terms of type $\partial_{\tau} \mathbf{A}_{\alpha}$ even such theories were studied in our previous works [33-41], see references therein.

The conditions $\mathbf{R}_{i a}=0$ and $\mathbf{R}_{a i}=0$ for the Ricci tensor $\operatorname{Ric}[\mathbf{D}]=\left\{\mathbf{R}_{\alpha \beta}=\left[\mathbf{R}_{i j}, \mathbf{R}_{i a}, \mathbf{R}_{a i}, \mathbf{R}_{a b}\right]\right\}$ are necessary if we want to keep the metric $\mathbf{g}(\tau)$ to be symmetric under nonholonomic Ricci flow evolution determined by (20). Geometric flow evolution and nonholonomic gravity of theories with nonsymmetric metrics were studied in [56], see references therein. In principle, we can work with any type normalization function $f$ which allows a general decoupling and integration of such systems of nonlinear PDEs. Such a normalization depends on frame and coordinate transforms and may encode (effective) cosmological constants, matter sources etc. We note that similar variational and/or geometric methods allows to derive from $\mathcal{W}(\tau)(17)$ certain types nonlinear evolution equations which are equivalent to (20). It is more difficult to solve explicitly such PDEs but a Wfunctional allows to elaborate directly on certain classes of thermodynamic models, see Sect. 5. 
3.3 Entropic gravity and gravitational field equations as Ricci solitons

For self-similar point $\tau=\tau_{0}$ configurations when $\partial_{\tau} \mathbf{g}_{\mu \nu}=$ 0 , with a corresponding choice of the normalizing geometric flow function $f$, the equations (20) transform into relativistic nonholonomic Ricci soliton equations

$$
\mathbf{R}_{i j}={ }^{t o t} \Upsilon_{i j}, \quad \mathbf{R}_{a b}={ }^{t o t} \Upsilon_{a b}, \quad \mathbf{R}_{i a}=\mathbf{R}_{a i}=0
$$

which are equivalent to (modified) Einstein equations in (MGT) GR for corresponding definitions of ${ }^{\text {tot }} \Upsilon_{\alpha \beta}$. A class of MGTs and GR can be formulated as geometric theories of entropic elastic origin which is similar to the idea of emergent gravity put forward by Verlinde [6,7], i.e. in the form (7) with (effective) entropic and quasiperiodic source ${ }^{\text {tot }} \Upsilon_{\alpha \beta}$ (15).

We conclude that an emergent gravity model in the Verlinde sense $[6,7,65]$, can be constructed for Lagrange distributions (14) and respective sources (15) introduced as generating data for the nonholonomic Hamilton equations (20) and respective relativistic Ricci solitons. Such geometric flow evolution theories and their spacetime elastic, quasiperiodic and thermodynamic properties are determined by the generalized $\mathcal{W}$-entropy (17).

\section{Decoupling and integrability of entropic flow equations}

In this section, we prove that the system of nonlinear PDEs (20) describing spacetime elastic and quasiperiodic flows and entropic gravity theories can be formally integrated in very general forms for generic off-diagonal metrics and canonical d-connections (in particular, for LC-configurations). The coefficients of geometric objects for such solutions depend on all spacetime coordinates via generating and integration functions and (effective) matter sources. The anholonomic frame deformation method, AFDM, for constructing exact solutions in MGTs and GR is developed for generating new classes of solutions encoding entropic quasiperiodic modifications in $\mathbf{g}(\tau)(2), \mathbf{D}(\tau)(4)$, and ${ }^{\text {tot }} \Upsilon_{\alpha \beta}(\tau)$ (15). For similar details and mathematical proofs, we refer readers to our previous works [55-60,63,64], on exact solutions in MGTs, and [33-41], for solutions with nonholonomic Ricci flows, and citations therein.

\subsection{Geometric flows with parametric modified Einstein equations}

Introducing effective sources, entropic geometric flow equations can written as modified Einstein equations with dependence on a temperature like parameter $\tau$. We show that such systems of nonlinear PDEs can be decoupled in general forms.

\subsubsection{Entropic quasiperiodic flow modifications of gravitational field equations}

Using nonholonomic frame transforms and tetradic (vierbein) fields, we introduce effective sources which in $\mathrm{N}$ adapted form are parameterized

$$
\begin{aligned}
\operatorname{eff} \Psi_{\mu \nu}(\tau) & =\mathbf{e}_{\mu}^{\mu^{\prime}}(\tau) \mathbf{e}_{v}{ }^{\nu^{\prime}}(\tau)\left[{ }^{\text {tot }} \Upsilon_{\mu^{\prime} v^{\prime}}(\tau)+\frac{1}{2} \partial_{\tau} \mathbf{g}_{\mu^{\prime} \nu^{\prime}}(\tau)\right] \\
& =\left[{ }_{h} ¥\left(\tau, x^{k}\right) \delta_{j}^{i}, ¥\left(\tau, x^{k}, y^{c}\right) \delta_{b}^{a}\right] .
\end{aligned}
$$

Such families of vielbein transforms $\mathbf{e}_{\mu^{\prime}}^{\mu}(\tau)=\mathbf{e}_{\mu^{\prime}}^{\mu}\left(\tau, u^{\gamma}\right)$ and their dual $\mathbf{e}_{v}^{v^{\prime}}\left(\tau, u^{\gamma}\right)$, when $\mathbf{e}^{\mu}=\mathbf{e}_{\mu^{\prime}}^{\mu} d u^{\mu^{\prime}}$ can be chosen for any frame/coordinate transforms of a $\mathrm{N}$-splitting structure (1). In result, the system of nonholonomic entropic R. Hamilton equations (20) can be written in the form (7) but with geometric objects depending additionally on a temperature like parameter $\tau$ and for effective source (22),

$\mathbf{R}_{\alpha \beta}(\tau)={ }^{e f f} ¥_{\alpha \beta}(\tau)$.

We note that such geometric evolution equations are for an undetermined normalization function $f(\tau)=f\left(\tau,\left(\tau, u^{\gamma}\right)\right.$ which can be defined explicitly for respective classes of exact or parametric solutions. For self-similar point $\tau=\tau_{0}$ configurations with $\partial_{\tau} \mathbf{g}_{\mu \nu}\left(\tau_{0}\right)=0$, this system of nonlinear PDEs transforms into the nonholonomic entropic Ricci soliton equations (21).

\subsubsection{Effective entropic sources for stationary and/or cosmological configurations}

The values $h^{¥}(\tau, x)$ and $¥(\tau, x, y)$ in (22) can be considered as generating data for (effective) matter sources. Prescribing such data, we impose certain nonholonomic frame constraints on geometric evolution and self-similar configurations of entropic and quasiperiodic structures. This type of $¥$-generating functions allows formal integrations of the system (23) in certain general forms.

Using frame transforms, the $\tau$-evolution of d-metric $\mathbf{g}(\tau)$ (2) can be parameterized for respective spherical symmetric coordinates $u^{\alpha}=\left(r, \theta, y^{3}=\varphi, t\right)$ or some cosmological coordinates $\left(x^{k}, y^{4}=t\right)$,

$g_{i}(\tau)=e^{\psi(\tau, r, \theta)}$,

$g_{a}(\tau)=\omega\left(\tau, r, \theta, y^{b}\right) h_{a}(\tau, r, \theta, \varphi)$,

$N_{i}^{3}(\tau)=w_{i}(\tau, r, \theta, \varphi), \quad N_{i}^{4}(\tau)=n_{i}(\tau, r, \theta, \varphi)$,

for $\omega=1$, stationary configurations;

$g_{i}(\tau)=e^{\psi\left(\tau, x^{k}\right)}, \quad g_{a}(\tau)=\omega\left(\tau, x^{k}, y^{b}\right) \bar{h}_{a}\left(\tau, x^{k}, t\right)$,

$N_{i}^{3}(\tau)=\bar{n}_{i}\left(\tau, x^{k}, t\right), \quad N_{i}^{4}(\tau)=\bar{w}_{i}\left(\tau, x^{k}, t\right)$, 
for $\omega=1$, cosmological configurations.

The AFDM results in more simple and explicit (still very general classes) of solutions if we work with nonholonomic configurations possessing at least one Killing symmetry, for instance, on $\partial_{4}=\partial_{t}$ for stationary solution or on $\partial_{3}=\partial_{\varphi}$, locally anisotropic solutions. ${ }^{11}$

We shall use brief notations of partial derivatives $\partial_{\alpha} q=$ $\partial q / \partial u^{\alpha}$ when a function $q\left(x^{k}, y^{a}\right)$,

$$
\begin{aligned}
& \partial_{1} q=q^{\bullet}=\partial q / \partial x^{1}, \quad \partial_{2} q=q^{\prime}=\partial q / \partial x^{2}, \\
& \partial_{3} q=\partial q / \partial y^{3}=\partial q / \partial \varphi=q^{\diamond}, \quad \partial_{4} q=\partial q / \partial t=\partial_{t} q=q^{*}, \\
& \partial_{33}^{2}=\partial^{2} q / \partial \varphi^{2}=\partial_{\varphi \varphi}^{2} q=q^{\diamond \diamond}, \quad \partial_{44}^{2}=\partial^{2} q / \partial t^{2}=\partial_{t t}^{2} q=q^{* *} .
\end{aligned}
$$

For respective Killing symmetries, the effective sources $¥(\tau, x, y)$ in (22) can be parameterized

$$
\begin{aligned}
\text { eff } & ¥{ }_{\nu}^{\mu}(\tau) \\
& = \begin{cases}{\left[{ }_{h} ¥(\tau, r, \theta) \delta_{j}^{i}, ¥(\tau, r, \theta, \varphi) \delta_{b}^{a}\right],} & \text { stationary configurations; } \\
\left.{ }_{h} \bar{¥}\left(\tau, x^{i}\right) \delta_{j}^{i}, \bar{¥}\left(\tau, x^{i}, t\right) \delta_{b}^{a}\right], & \text { cosmological configurations. }\end{cases}
\end{aligned}
$$

Considering as typical examples two types of a Killing space symmetry or time like Killing symmetry for effective generating sources, we shall construct and study properties of two general classes of exact solutions (the first one will be for stationary configurations which may contain $\mathrm{BH}$ solutions and the second one will be for cosmological type solutions).

\subsection{Nontrivial Ricci d-tensors and decoupling of entropic flow equations}

In this subsection, we outline the key steps for proofs of general decoupling and integrability of (modified) Einstein equations with effective sources (26).

\subsubsection{Off-diagonal metric ansatz, (non) holonomic variables, and ODEs and PDEs}

Let us summarize in Table 1 below the data on nonholonomic $3+1$ and $2+2$ variables and corresponding ansatz which allows to transform geometric and entropic flow equations and, a nonholonomic Ricci solitons, gravitational field equations in entropic MGTs and GR into respective systems of nonlinear ordinary differential equations, ODEs, and partial differential equations, PDEs. All formulas will be proven in next subsections. We model a nonholonomic deformation

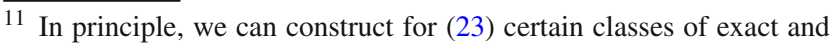
parametric off-diagonal solutions generically depending on all spacetime coordinates $\left(x^{k}, y^{a}\right)$ but that would result in hundreds of pages with a cumbersome formulas for respective geometric techniques, see [55-59] and references therein.
}

with $\eta$-polarization functions, $\stackrel{\mathrm{g}}{\rightarrow} \mathbf{g}(\tau)$, of a 'prime' metric, $\stackrel{\circ}{\mathbf{g}}$, into a family 'target' d-metrics $\mathbf{g}(\tau)(2)$, if

$$
\begin{aligned}
\mathbf{g}(\tau)= & \eta_{i}\left(\tau, x^{k}\right) \stackrel{\circ}{g}_{i} d x^{i} \otimes d x^{i} \\
& +\eta_{a}\left(\tau, x^{k}, y^{b}\right) \stackrel{\circ}{h}_{a} \mathbf{e}^{a}[\eta] \otimes \mathbf{e}^{a}[\eta],
\end{aligned}
$$

where the target $\mathrm{N}$-elongated basis is determined by $N_{i}^{a}(\tau, u)$ $=\eta_{i}^{a}\left(\tau, x^{k}, y^{b}\right) \stackrel{\circ}{N}_{i}^{a}\left(\tau, x^{k}, y^{b}\right)$ in the form ${ }^{12} \mathbf{e}^{\alpha}[\eta]=\left(d x^{i}, \mathbf{e}^{a}=\right.$ $\left.d y^{a}+\eta_{i}^{a} \stackrel{\circ}{N}_{i}^{a} d x^{i}\right)$. The values $\eta_{i}(\tau)=\eta_{i}\left(\tau, x^{k}\right), \eta_{a}(\tau)=$ $\eta_{a}\left(\tau, x^{k}, y^{b}\right)$ and $\eta_{i}^{a}(\tau)=\eta_{i}^{a}\left(\tau, x^{k}, y^{b}\right)$ are called respectively geometric/entropic flow or gravitational polarization functions, or $\eta$-polarizations. Any $\mathbf{g}(\tau)$ is subjected to the condition that it defines a solution of modified Einstein equations resulting in entropic quasiperiodic geometric flows and/or via nonholonomic deformations. A general prime metric in a coordinate parametrization is of type $\stackrel{\circ}{\mathbf{g}}=$ $\stackrel{\circ}{g}_{\alpha \beta}\left(x^{i}, y^{a}\right) d u^{\alpha} \otimes d u^{\beta}$, which can be also represented equivalently in $\mathrm{N}$-adapted form

$$
\begin{gathered}
\stackrel{\circ}{\mathbf{g}}=\stackrel{\circ}{g}_{\alpha}(u) \stackrel{\circ}{\mathbf{e}}^{\alpha} \otimes \stackrel{\circ}{\mathbf{e}}^{\beta}=\stackrel{\circ}{g}_{i}(x) d x^{i} \otimes d x^{i}+\stackrel{\circ}{g}_{a}(x, y) \stackrel{\circ}{\mathbf{e}}^{a} \otimes \stackrel{\circ}{\mathbf{e}}^{a}, \\
\text { for } \stackrel{\circ}{\mathbf{e}}^{\alpha}=\left(d x^{i}, \mathbf{e}^{a}=d y^{a}+\stackrel{\circ}{N}_{i}^{a}(u) d x^{i}\right), \quad \text { and } \\
\stackrel{\circ}{\alpha}_{\alpha}=\left(\check{\mathbf{e}}_{i}=\partial / \partial y^{a}-\stackrel{\circ}{N}_{i}^{b}(u) \partial / \partial y^{b}, e_{a}=\partial / \partial y^{a}\right) .
\end{gathered}
$$

Such a d-metric can be, or not, a solution of some gravitational field equations in a MGT or GR but it nonholonomic deformations to a target metric (27) are subjected to the condition to define an exact or parametric solutions of certain entropic flow evolutions equations.

In our works, we are interested usually in two physically important cases when $\mathbf{g}$ (28) defines a BH solution (for instance, a vacuum Kerr, or Schwarzschild, Kerr-(anti) de Sitter metric), or a Friedman-Lemaître-Robertson-Walker (FLRW) type metric, or any Bianchi anisotropic metrics. For diagonalizable prime metrics (the off-diagonal structure of the Kerr metric is determined by rotation frames and coordinates), we can always find a coordinate system when $\stackrel{\circ}{N}_{i}^{b}=0$. To avoid nonholonomic deformations with singular coordinates is convenient to construct exact solutions with nontrivial functions $\eta_{\alpha}=\left(\eta_{i}, \eta_{a}\right), \eta_{i}^{a}$, and nonzero coefficients $\stackrel{\circ}{N}_{i}^{b}(u)$. We have to consider necessary type frame/coordinate transforms. For a d-metric (27), we can analyze the conditions of existence and geometric/physical properties of some target and/or prime solutions, for instance, when $\eta_{\alpha} \rightarrow 1$ and $N_{i}^{a} \rightarrow \stackrel{\circ}{N}_{i}^{a}$. The values $\eta_{\alpha}=1$ and/or $\stackrel{\circ}{N}_{i}^{a}=0$ can be imposed as some special nonholonomic constraints. ${ }^{13}$ In brief, we shall denote certain nonholonomic

\footnotetext{
${ }^{12}$ We do not consider summation on repeating indices if they are not written as contraction of "up-low" ones.

${ }^{13}$ We can consider flow evolution of a physical important target metric $\mathbf{g}$ (27) with generic off-diagonal terms as an "almost" BH, or FLRW cosmological, like metric. Such parametric solutions are constructed for small nonholonomic deformations on some constant parameters $\eta_{\alpha}=\left(\eta_{i}, \eta_{a}\right), \eta_{i}^{a}$, for $0 \leq \varepsilon_{\alpha}, \varepsilon_{i}^{b} \ll 1$, when $\eta_{i} \simeq$
} 
Table 1 Entropic quasiperiodic flow modified Einstein eqs as systems of nonlinear PDEs and the Anholonomic Frame Deformation Method, AFDM, for constructing generic off-diagonal exact, parametric, and physically important solutions

\section{Diagonal ansatz: PDEs $\rightarrow$ ODEs}

Radial coordinates $u^{\alpha}=(r, \theta, \varphi, t)$

LC-connection $\stackrel{\circ}{\nabla}$

Diagonal ansatz $g_{\alpha \beta}(u)$

$$
=\left(\begin{array}{llll}
\stackrel{\circ}{g}_{1} & & & \\
& \stackrel{\circ}{g}_{2} & & \\
& & \stackrel{\circ}{g}_{3} & \\
& & & \stackrel{\circ}{g}_{4}
\end{array}\right)
$$

$\stackrel{\circ}{g}_{\alpha \beta}= \begin{cases}\stackrel{\circ}{g}_{\alpha}(r) & \text { for BHs } \\ \stackrel{\circ}{g}_{\alpha}(t) & \text { for FLRW }\end{cases}$

Coord.tranfsorms $e_{\alpha}=e_{\alpha}^{\alpha^{\prime}} \partial_{\alpha^{\prime}}$,

$e^{\beta}=e_{\beta^{\prime}}^{\beta} d u^{\beta^{\prime}}, \stackrel{\circ}{g}_{\alpha \beta}=\stackrel{\circ}{g}_{\alpha^{\prime} \beta^{\prime}} e_{\alpha}^{\alpha^{\prime}} e_{\beta}^{\beta^{\prime}}$

$\stackrel{\circ}{\mathbf{g}}_{\alpha}\left(x^{k}, y^{a}\right) \rightarrow \stackrel{\circ}{g}_{\alpha}(r)$, or $\stackrel{\circ}{g}_{\alpha}(t)$,

$\stackrel{\circ}{N}_{i}^{a}\left(x^{k}, y^{a}\right) \rightarrow 0$.

$\stackrel{\circ}{\nabla}, R i c=\left\{\stackrel{\circ}{R}_{\beta \gamma}\right\}$

${ }^{m} \mathcal{L}[\phi] \rightarrow{ }^{m} \mathbf{T}_{\alpha \beta}[\phi]$

Trivial equations for $\stackrel{\circ}{\nabla}$-torsion
[Coord.frames]

[N-adapt. fr.]

$$
u=(x, y):
$$

[Connections]

Ricci tensors

Sources

LC-conditions

\section{AFDM: PDEs with decoupling; generating functions}

$2+2$ splitting, $u^{\alpha}=\left(x^{1}, x^{2}, y^{3}, y^{4}=t\right)$; flow parameter $\tau$

$\mathbf{N}: T \mathbf{V}=h T \mathbf{V} \oplus v T \mathbf{V}$, locally $\mathbf{N}=\left\{N_{i}^{a}(x, y)\right\}$

canonical connection distortion $\mathbf{D}=\nabla+\mathbf{Z}$

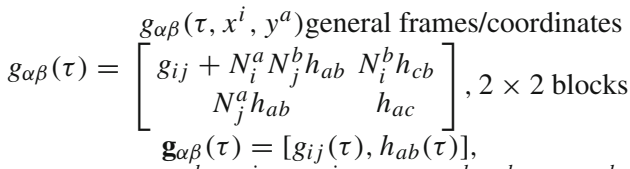

$\mathbf{g}(\tau)=\mathbf{g}_{i}\left(\tau, x^{k}\right) d x^{i} \otimes d x^{i}+\mathbf{g}_{a}\left(\tau, x^{k}, y^{b}\right) \mathbf{e}^{a} \otimes \mathbf{e}^{b}$

$g_{\alpha \beta}(\tau)= \begin{cases}g_{\alpha \beta}\left(\tau, r, \theta, y^{3}=\varphi\right) & \text { stationary configurations } \\ g_{\alpha \beta}\left(\tau, r, \theta, y^{4}=t\right) & \text { cosm. configurations }\end{cases}$

$\left\{\begin{array}{l}\mathbf{g}_{i}(\tau, r, \theta), \mathbf{g}_{a}(\tau, r, \theta, \varphi), \\ \text { or } \mathbf{g}_{i}(\tau, r, \theta), \mathbf{g}_{a}(\tau, r, \theta, t), \\ N_{i}^{3}(\tau)=w_{i}(\tau, r, \theta, \varphi), N_{i}^{4}=n_{i}(\tau, r, \theta, \varphi), \\ \text { or } N_{i}^{3}(\tau)=n_{i}(\tau, r, \theta, t), N_{i}^{4}=w_{i}(\tau, r, \theta, t),\end{array}\right.$

d-metrics

$\mathbf{D}, \mathcal{R} i c=\left\{\mathbf{R}_{\beta \gamma}\right\}$

$$
\boldsymbol{\Upsilon}_{\nu}^{\mu}(\tau)=\mathbf{e}_{\mu^{\prime}}^{\mu} \mathbf{e}_{v}^{v^{\prime}} \boldsymbol{\Upsilon}_{v^{\prime}}^{\mu^{\prime}}
$$

$=\operatorname{diag}\left[{ }_{h} ¥\left(\tau, x^{i}\right) \delta_{j}^{i}, ¥\left(\tau, x^{i}, \varphi\right) \delta_{b}^{a}\right]$, stationary conf.

$=\operatorname{diag}\left[h \bar{¥}\left(\tau, x^{i}\right) \delta_{j}^{i}, \bar{¥}\left(\tau, x^{i}, t\right) \delta_{b}^{a}\right]$, cosmol. conf. entropic deformations of a prime d-metrics into a target one as $\stackrel{\circ}{\mathbf{g}} \rightarrow \mathbf{g}=\left[g_{\alpha}=\eta_{\alpha} \stackrel{\circ}{g}_{\alpha}, \eta_{i}^{a} \stackrel{\circ}{i}_{i}^{a}\right]$.

Table 1 outlines the key steps for developing the AFDM to theories of entropic quasiperiodic geometric flows. In this work, the formulas depend on a flow temperature like parameter $\tau$ and the constructions are for effective matter sources encoding entropic nonholonomic flows and deformations.

\subsubsection{Cosmological Ricci d-tensors, LC-conditions, and nonlinear symmetries}

For locally anisotropic cosmological configurations, we can consider geometric data when coefficients of the geometric objects do not depend on a space like $y^{3}$ with respect to certain classes of $\mathrm{N}$-adapted frames. Using d-metric data (25) with $\omega=1$ and a source $\left[h \bar{\Psi}\left(\tau, x^{i}\right), \bar{¥}\left(\tau, x^{i}, t\right)\right]$ (26), we write the entropic flow modified Einstein equations (23) in

Footnote 13 continued

$\check{\eta}_{i}\left(\tau, x^{k}\right)\left[1+\varepsilon_{i} \chi_{i}\left(\tau, x^{k}\right)\right] \simeq 1+\varepsilon_{i} \chi_{i}\left(\tau, x^{k}\right), \eta_{a} \simeq \check{\eta}_{a}\left(\tau, x^{k}, y^{b}\right)[1+$ $\left.\varepsilon_{a} \chi_{a}\left(\tau, x^{k}, y^{b}\right)\right] \simeq 1+\varepsilon_{a} \chi_{a}\left(\tau, x^{k}, y^{b}\right)$, and $\eta_{i}^{a} \simeq \check{\eta}_{i}^{a}\left(\tau, x^{k}, y^{b}\right)[1+$ $\left.\varepsilon_{i}^{a} \chi_{i}^{a}\left(\tau, x^{k}, y^{b}\right)\right] \simeq 1+\varepsilon_{i}^{a} \chi_{i}^{a}\left(\tau, x^{k}, y^{b}\right)$. Parametric $\varepsilon$-decompositions can be performed in a self-consistent form by omitting quadratic and higher terms after a class of solutions have been found for some evolution or nonholonomic deformation data $\left(\eta_{\alpha}, \eta_{i}^{a}\right)$. For certain subclasses of solutions, we can consider that $\varepsilon_{i}, \varepsilon_{a}, \varepsilon_{i}^{a} \sim \varepsilon$, when only one small parameter is considered for all coefficients of nonholonomic deformations. We can work with mixed types of solutions and model only small diagonal deformations $\varepsilon_{i}, \varepsilon_{a}, \sim \varepsilon$ of metrics, for some general $\eta_{i}^{a}$. Alternatively, we consider nontrivial $\eta_{\alpha}$ but $\varepsilon_{i}^{a} \sim \varepsilon$. the form ${ }^{14}$ :

$$
\begin{aligned}
\mathbf{R}_{1}^{1}(\tau)= & \mathbf{R}_{2}^{2}(\tau)=-{ }_{h} \bar{¥}(\tau) \text { i.e. } \frac{g_{1}^{\bullet} g_{2}^{\bullet}}{2 g_{1}}+\frac{\left(g_{2}^{\bullet}\right)^{2}}{2 g_{2}} \\
& -g_{2}^{\bullet \bullet}+\frac{g_{1}^{\prime} g_{2}^{\prime}}{2 g_{2}}+\frac{\left(g_{1}^{\prime}\right)^{2}}{2 g_{1}} \\
& -g_{1}^{\prime \prime}=-2 g_{1} g_{2 h} \bar{¥} ; \\
\mathbf{R}_{3}^{3}(\tau)= & \mathbf{R}_{4}^{4}(\tau)=-\bar{¥}(\tau) \text { i.e. } \frac{\left(\bar{h}_{3}^{*}\right)^{2}}{2 \bar{h}_{3}}+\frac{\bar{h}_{3}^{*} \bar{h}_{4}^{*}}{2 \bar{h}_{4}} \\
& -\bar{h}_{3}^{* *}=-2 \bar{h}_{3} \bar{h}_{4} \bar{F}_{j} ; \\
& \bar{h}_{3} \bar{n}_{3 k}^{* *}+\left(\frac{3}{2} \bar{h}_{3}^{*}-\frac{\bar{h}_{3}}{\bar{h}_{4}} \bar{h}_{4}^{*}\right) \frac{\bar{n}_{k}^{*}}{2 \bar{h}_{4}}=0 ; \\
2 \bar{h}_{3} \mathbf{R}_{4 k}(\tau)= & -\bar{w}_{k}\left[\frac{\left(\bar{h}_{3}^{*}\right)^{2}}{2 \bar{h}_{3}}+\frac{\bar{h}_{3}^{*} \bar{h}_{4}^{*}}{2 \bar{h}_{4}}-\bar{h}_{3}^{* *}\right] \\
& +\frac{\bar{h}_{3}^{*}}{2}\left(\frac{\partial_{k} \bar{h}_{3}}{\bar{h}_{3}}+\frac{\partial_{k} \bar{h}_{4}}{\bar{h}_{4}}\right)-\partial_{k} \bar{h}_{3}^{*}=0 .
\end{aligned}
$$

This system of nonlinear PDEs can be transformed, respectively, into a system of equation for stationary configurations

\footnotetext{
14 There are considered partial derivatives $\partial_{t} q=\partial_{4} q=q^{*}$ and $\partial_{i} q=$ $\left(\partial_{1} q=q^{\bullet}, \partial_{2} q=q^{\prime}\right)$; we use over-lined symbols in order to emphasize that certain values are not stationary but depend on a time like coordinate $t$.
} 
if

${ }_{h} \bar{\Upsilon}\left(\tau, x^{i}\right) \rightarrow{ }_{h} \Upsilon\left(\tau, x^{i}\right), \quad \bar{\Upsilon}\left(\tau, x^{i}, t\right) \rightarrow \Upsilon\left(\tau, x^{i}, y^{3}=\varphi\right)$,

$\bar{h}_{3}\left(\tau, x^{i}, t\right) \rightarrow h_{4}\left(\tau, x^{i}, \varphi\right), \quad \bar{h}_{4}\left(\tau, x^{i}, t\right) \rightarrow h_{3}\left(\tau, x^{i}, \varphi\right)$,

$\bar{h}_{3}^{*}\left(\tau, x^{i}, t\right) \rightarrow h_{4}^{\diamond}\left(\tau, x^{i}, \varphi\right)$,

$\bar{h}_{4}^{*}\left(\tau, x^{i}, t\right) \rightarrow h_{3}^{\diamond}\left(\tau, x^{i}, \varphi\right), \quad \bar{w}_{k}\left(\tau, x^{i}, t\right) \rightarrow n_{k}\left(\tau, x^{i}, \varphi\right)$,

$\bar{n}_{k}\left(\tau, x^{i}, t\right) \rightarrow w_{k}\left(\tau, x^{i}, \varphi\right)$ etc.

Such a duality exists for Lorentz manifolds with a Killing symmetry when $y^{3}$ is a space like coordinate and $y^{4}=t$ is a time like coordinated. This duality simplifies various applications of the AFDM when we can redefine the procedure considered in the previous subsection for stationary nonholonomic configurations to certain time dependent ones. It allows use to prove decoupling properties and generate cosmological like solutions if there are known certain stationary configurations, or inversely to find certain stationary metrics as analogs of corresponding cosmological ones. ${ }^{15}$

We can rewrite the nonlinear PDE (29)-(32) in an explicit decoupled form if we introduce the coefficients $\bar{\alpha}_{i}=$ $\left(\partial_{t} \bar{h}_{3}\right)\left(\partial_{i} \bar{\varpi}\right), \bar{\beta}=\left(\partial_{t} \bar{h}_{3}\right)\left(\partial_{t} \bar{\varpi}\right), \bar{\gamma}=\partial_{t}\left(\ln \left|\bar{h}_{3}\right|^{3 / 2} /\left|\bar{h}_{4}\right|\right)$, where $\bar{\varpi}=\ln \mid \partial_{t} \bar{h}_{3} / \sqrt{\left|\bar{h}_{3} \bar{h}_{4}\right|}$. For $\partial_{t} h_{a} \neq 0$ and $\partial_{t} \varpi \neq 0$, we obtain such equations

$$
\begin{gathered}
\psi^{\bullet \bullet}+\psi^{\prime \prime}=2_{h} \bar{\mp} ; \quad \bar{\varpi}^{*} \bar{h}_{3}^{*}=2 \bar{h}_{3} \bar{h}_{4} \bar{¥} ; \quad \bar{n}_{i}^{* *}+\bar{\gamma}_{i}^{*}=0 ; \\
\bar{\beta} \bar{w}_{i}-\bar{\alpha}_{i}=0 .
\end{gathered}
$$

We can integrate such equations "step by step" for any generating function $\bar{\Psi}\left(x^{i}, t\right):=e^{\bar{\varpi}}$ and sources ${ }_{h} \bar{\Upsilon}\left(x^{i}\right)$ and $\bar{\Upsilon}\left(x^{k}, t\right)$, see next subsection.

Nonlinear symmetries for generating functions and sources with effective cosmological constant: The system (34) with respective coefficients relates four functions $\left(\bar{h}_{3}, \bar{h}_{4}, \bar{\Upsilon}, \bar{\Psi}\right)$ when a very important nonlinear symmetry for locally anisotropic cosmological solutions and respective generating functions, $(\bar{\Psi}(\tau), \bar{\Upsilon}(\tau)) \Longleftrightarrow(\bar{\Phi}(\tau), \Lambda(\tau))$ can be found,

$$
\begin{aligned}
\bar{\Lambda}\left(\bar{\Psi}^{2}\right)^{*}= & |\bar{¥}|\left(\bar{\Phi}^{2}\right)^{*}, \text { or } \overline{\Lambda \Psi}^{2}=\bar{\Phi}^{2}|\bar{¥}| \\
& -\int d t \bar{\Phi}^{2}|\bar{¥}|^{*} .
\end{aligned}
$$

This nonlinear symmetry allows us to introduce a new generating function $\bar{\Phi}\left(x^{i}, t\right)$ and an (effective) cosmological constant $\bar{\Lambda}(\tau) \neq 0$, which can be applied both for generating exact off-diagonal solutions in explicit forms and elaborating on locally anisotropic cosmological scenarios with cosmological constants, for instance, considered in entropic gravity.

\footnotetext{
15 The LC-conditions (8) for stationary configurations transform into equations with coefficients depending on $t, \partial_{t} \bar{w}_{i}=\left(\partial_{i}-\right.$ $\left.\bar{w}_{i} \partial_{t}\right) \ln \sqrt{\left|\bar{h}_{4}\right|},\left(\partial_{i}-\bar{w}_{i} \partial_{t}\right) \ln \sqrt{\left|\bar{h}_{3}\right|}=0, \partial_{k} \bar{w}_{i}=\partial_{i} \bar{w}_{k}, \partial_{t} \bar{n}_{i}=$ $0, \partial_{i} \bar{n}_{k}=\partial_{k} \bar{n}_{i}$. Such nonlinear first order PDEs containing $\partial_{t}$ can be solved in explicit form for certain classes of additional nonholonomic constraints on cosmological d-metrics and N-coefficients, see (25).
}

4.3 Integrability of entropic quasiperiodic geometric flow equations

We generate and study geometric properties of two classes of generic off-diagonal solutions with elasticity and quasiperiodic structures of the system of nonlinear PDEs (23). The first one is for stationary configurations and the second one is considered for locally anisotropic cosmological models.

\subsubsection{Off-diagonal cosmological solutions with elastic quasiperiodic structures}

Applying the AFDM, we can construct cosmological solutions (which, in general, are locally anisotropic) of the entropic flow modified Einstein equations (23) with $\mathrm{N}$ adapted sources $_{h} \bar{¥}(\tau)={ }_{h} \bar{¥}\left(\tau, x^{k}\right)$ and $\bar{¥}(\tau)=\bar{¥}\left(\tau, x^{k}, t\right)$, see parameterizations for cosmological configurations in (26). Integrating "step by step" the system of the nonlinear PDEs (29)-(32) decoupled in the form (34), we obtain such d-metric coefficients for (2),

$$
\begin{aligned}
g_{i}(\tau)= & e^{\psi\left(\tau, x^{k}\right)} \text { as a solution of 2-d Poisson eqs. } \psi^{\bullet \bullet}+\psi^{\prime \prime} \\
= & 2_{h} \bar{\Psi}(\tau) ; \\
g_{3}(\tau)= & \bar{h}_{3}\left(\tau, x^{i}, t\right)=h_{3}^{[0]}\left(\tau, x^{k}\right) \\
& -\int d t \frac{\left(\bar{\Psi}^{2}\right)^{*}}{4 \bar{\Psi}}=h_{3}^{[0]}\left(\tau, x^{k}\right)-\bar{\Phi}^{2} / 4 \bar{\Lambda}(\tau) ; \\
g_{4}(\tau)= & \bar{h}_{4}\left(\tau, x^{i}, t\right)=-\frac{\left(\bar{\Psi}^{2}\right)^{*}}{4 \bar{\Psi}^{2} \bar{h}_{3}} \\
= & -\frac{\left.\bar{\Psi}^{2}\right)^{*}}{4 \bar{\Psi}^{2}\left(h_{3}^{[0]}\left(\tau, x^{k}\right)-\int d t\left(\bar{\Psi}^{2}\right)^{*} / 4 \bar{\Psi}\right)} \\
= & -\frac{\left[\left(\bar{\Phi}^{2}\right)^{\diamond}\right]^{2}}{4 \bar{h}_{3}\left|\bar{\Lambda}(\tau) \int d t \bar{\Psi}^{2}\left[\bar{\Phi}^{2}\right]^{\diamond}\right|} \\
= & -\frac{\left[\left(\bar{\Phi}^{2}\right)^{*}\right]^{2}}{4\left[h_{3}^{[0]}\left(x^{k}\right)-\bar{\Phi}^{2} / 4 \bar{\Lambda}(\tau)\right]\left|\int d t \bar{\Psi}\left[\bar{\Phi}^{2}\right]^{*}\right|} .
\end{aligned}
$$

The N-connection coefficients are computed,

$$
\begin{aligned}
N_{k}^{3}(\tau)= & \bar{n}_{k}\left(\tau, x^{i}, t\right)={ }_{1} n_{k}\left(\tau, x^{i}\right)+{ }_{2} n_{k}\left(\tau, x^{i}\right) \\
& \times \int d t \frac{\left(\bar{\Psi}^{*}\right)^{2}}{\bar{\Psi}^{2}\left|h_{3}^{[0]}\left(\tau, x^{i}\right)-\int d t\left(\bar{\Psi}^{2}\right)^{*} / 4 \bar{\Psi}\right|^{5 / 2}} \\
= & { }_{1} n_{k}\left(\tau, x^{i}\right)+{ }_{2} n_{k}\left(\tau, x^{i}\right) \\
& \times \int d t \frac{\left(\bar{\Phi}^{*}\right)^{2}}{4\left|\bar{\Lambda}(\tau) \int d t \bar{¥}\left[\bar{\Phi}^{2}\right]^{*}\right|\left|\bar{h}_{3}\right|^{5 / 2}} ; \\
N_{i}^{4}(\tau)= & \bar{w}_{i}\left(\tau, x^{i}, t\right)=\frac{\partial_{i} \bar{\Psi}}{\bar{\Psi}^{*}}=\frac{\partial_{i} \bar{\Psi}^{2}}{\left(\bar{\Psi}^{2}\right)^{*}}=\frac{\partial_{i}\left[\int d t \bar{¥}\left(\bar{\Phi}^{2}\right)^{*}\right]}{\bar{\Psi}\left(\bar{\Phi}^{2}\right)^{*}},
\end{aligned}
$$


In these formulas, $h_{3}^{[0]}\left(\tau, x^{k}\right),{ }_{1} n_{k}\left(\tau, x^{i}\right)$, and ${ }_{2} n_{k}\left(\tau, x^{i}\right)$ are integration functions encoding various possible sets of (non) commutative parameters and integration constants running on $\tau$ for geometric evolution flows. We can chose different generating data $\left(\bar{\Psi}\left(\tau, x^{i}, t\right), \bar{\Upsilon}\left(\tau, x^{i}, t\right)\right)$ or $\left(\bar{\Phi}\left(\tau, x^{i}, t\right), \bar{\Lambda}(\tau)\right)$ which are related by nonlinear differential/integral transforms (35), and respective integration functions. Such values should be chosen in explicit form following certain topology/symmetry/asymptotic conditions for some classes of exact/parametric cosmological solutions. The coefficients (36) and (37) define generic off-diagonal cosmological solutions if the corresponding anholonomy coefficients are not trivial. Such locally cosmological solutions are with nontrivial nonholonomically induced d-torsion and $\mathrm{N}$-adapted coefficients which can be computed in explicit form. In order to generate as particular cases some well-known cosmological FLRW, or Bianchi, type metrics, we have to consider data of type $(\bar{\Psi}(\tau, t), \bar{\Upsilon}(\tau, t))$, or $(\bar{\Phi}(\tau, t), \bar{\Lambda}(\tau))$, with integration functions which allow frame/ coordinate transforms to respective (off-) diagonal configurations $g_{\alpha \beta}(\tau, t)$.

Let us analyze certain important nonholonomic evolution properties of above locally anisotropic cosmological solutions using the formulas for effective sources (22) with cosmological parameterizations (26). In $\mathrm{N}$-adapted form, we obtain a system of equations with first order evolution derivatives $\partial_{\tau}$ when the v-part of vierbeinds depend on a time like coordinate $y^{4}=t, \mathbf{e}_{\mu}^{\mu^{\prime}}(\tau)=$ $\left[\mathbf{e}_{1}^{1^{\prime}}\left(\tau, x^{k}\right), \mathbf{e}_{2}^{2^{\prime}}\left(\tau, x^{k}\right), \mathbf{e}_{3}^{3^{\prime}}\left(\tau, x^{k}, t\right), \mathbf{e}_{4}^{4^{\prime}}\left(\tau, x^{k}, t\right)\right] ;$ there are considered coordinates $\left(x^{k}, t\right)$, when the dependence on $y^{3}$ can be omitted because of Killing symmetry on $\partial_{3}$. We can consider frame transforms for generating effective sources, $\left.{ }^{e f f} ¥_{i}(\tau)=\left[\mathbf{e}_{i}^{i^{\prime}}(\tau)\right]^{2}\right)\left[{ }^{t o t} \boldsymbol{\Upsilon}_{i^{\prime} 1^{\prime}}(\tau)+\frac{1}{2} \partial_{\tau} \mathbf{g}_{1^{\prime}}(\tau)\right]=$ ${ }_{h} ¥\left(\tau, x^{k}\right)$,

$$
\begin{aligned}
\operatorname{eff}_{¥_{a}(\tau)} & \left.=\left[\mathbf{e}_{a}^{a^{\prime}}(\tau)\right]^{2}\right)\left[{ }^{t o t} \Upsilon_{a^{\prime} a^{\prime}}(\tau)+\frac{1}{2} \partial_{\tau} \mathbf{g}_{a^{\prime}}(\tau)\right] \\
& =¥\left(\tau, x^{k}, t\right) .
\end{aligned}
$$

In these formulas, we can prescribe any values for the matter sources ${ }^{t o t} \boldsymbol{\Upsilon}_{\mu \nu}(\tau)$ in a cosmological or spacetime QC model. Then, for simplicity, we can consider $\mathrm{N}$-adapted diagonal configurations and integrate on $\tau$ and determine a cosmological evolution flow of $\mathbf{g}_{\alpha^{\prime}}\left(\tau, x^{k}, t\right)$ modelled as a nonholonomic and nonlinear geometric diffusion process. All geometric constructions are performed with respect to a new system of reference determined by $\mathbf{e}_{\mu}^{\mu^{\prime}}\left(\tau, x^{k}, t\right)$. We have to prescribe some locally anisotropic generating values $\left[\mathbf{e}_{\mu}^{\mu^{\prime}}\left(\tau, x^{k}, t\right),{ }^{\text {tot }} \boldsymbol{\Upsilon}_{\mu \nu}\left(\tau, x^{k}, t\right)\right]$ which are compatible with certain observational data, for instance, in modern cosmology and dark matter and dark energy physics.

\subsubsection{Quadratic line elements for off-diagonal cosmological configurations with elastic flows}

Any coefficient $\bar{h}_{3}(\tau)=\bar{h}_{3}\left(\tau, x^{k}, t\right)=h_{3}^{[0]}\left(\tau, x^{k}\right)-$ $\bar{\Phi}^{2} / 4 \bar{\Lambda}(\tau), \bar{h}_{3}^{*} \neq 0$, can be considered also as a generating function, for instance, for entropic quasiperiodic configurations. Using formulas (36), we find $\bar{\Phi}^{2}=-4 \bar{\Lambda}(\tau) \bar{h}_{3}(\tau, r, \theta, t)$ transforming (35) in $\left(\bar{\Psi}^{2}\right)^{*}=\int d t \bar{¥}_{3}^{*}$. Introducing such values into the formulas for $\bar{h}_{a}$ and $\bar{¥}$ in (36) and (37), we construct locally anisotropic cosmological solutions parameterized by d-metrics (2) with $\mathrm{N}$-adapted coefficients (25),

$$
\begin{aligned}
& d s^{2}=e^{\psi\left(\tau, x^{k}\right)}\left[\left(d x^{1}\right)^{2}+\left(d x^{2}\right)^{2}\right] \\
& \left\{\begin{array}{l}
\bar{h}_{3}\left[d y^{3}+\left({ }_{1} n_{k}+4_{2} n_{k} \int d t \frac{\left(\bar{h}_{3}^{*}\right)^{2}}{\left|\int d y^{4} \bar{¥}_{3}^{*}\right|\left(\bar{h}_{3}\right)^{5 / 2}}\right) d x^{k}\right. \\
-\frac{\left(\bar{h}_{3}^{*}\right)^{2}}{\left|\int d t \bar{¥} \bar{h}_{3}^{*}\right| \bar{h}_{3}}\left[d t+\frac{\partial_{i}\left(\int d t \bar{¥} \bar{h}_{3}^{*}\right)}{\bar{¥} \bar{h}_{3}^{*}} d x^{i}\right]
\end{array}\right. \\
& \text { or }
\end{aligned}
$$

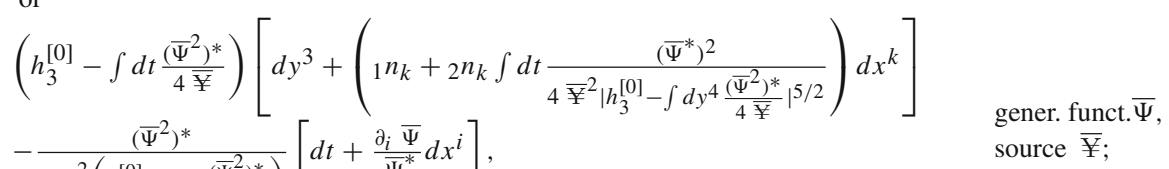

$$
\begin{aligned}
& + \begin{cases}-\frac{\left(\bar{\Psi}^{2}\right)^{*}}{4 \bar{\Psi}^{2}\left(h_{3}^{[0]}-\int d t \frac{\left(\bar{\Psi}^{2}\right)^{*}}{4 \bar{\Psi}}\right)}\left[d t+\frac{\partial_{i} \bar{\Psi}}{\bar{\Psi}^{*}} d x^{i}\right], & \text { gener. fun } \\
\text { source } \bar{¥} ;\end{cases} \\
& \text { or } \\
& \left(h_{3}^{[0]}-\frac{\bar{\Phi}^{2}}{4 \bar{\Lambda}}\right)\left[d y^{3}+\left({ }_{1} n_{k}+{ }_{2} n_{k} \int d t \frac{\left[\left(\bar{\Phi}^{2}\right)^{*}\right]^{2}}{\left|4 \bar{\Lambda} \int d y^{4} \bar{¥}\left(\bar{\Phi}^{2}\right)^{*}\right|}\left|h_{4}^{[0]}\left(x^{k}\right)-\frac{\Phi^{2}}{4 \bar{\Lambda}}\right|^{-5 / 2}\right) d x^{k}\right] \\
& -\frac{\left[\left(\bar{\Phi}^{2}\right)^{*}\right]^{2}}{\left|4 \bar{\Lambda} \int d y^{4} \bar{\Psi}\left(\bar{\Phi}^{2}\right)^{*}\right|\left(h_{3}^{[0]}-\frac{\Phi^{2}}{4 \bar{\Lambda}}\right)}\left[d t+\frac{\partial_{i}\left[\int d t \bar{¥}\left(\bar{\Phi}^{2}\right)^{*}\right]}{\bar{\Psi}\left(\bar{\Phi}^{2}\right)^{*}} d x^{i}\right] \text {, }
\end{aligned}
$$


Such solutions posses a Killing symmetry on $\partial_{3}$ and can be re-written in terms of $\eta$-polarization function functions for target locally anisotropic cosmological metrics $\widehat{\mathbf{g}}=\left[g_{\alpha}=\right.$ $\left.\eta_{\alpha} \stackrel{\circ}{g}_{\alpha}, \eta_{i}^{a} \stackrel{\circ}{N}_{i}^{a}\right]$ encoding primary cosmological data $\left[\stackrel{\circ}{g}_{\alpha}, \stackrel{\circ}{N}_{i}^{a}\right]$.

\subsubsection{Off-diagonal Levi-Civita entropic and quasiperiodic cosmological configurations}

We can extract and model entropic flow evolution of cosmological spacetimes in GR. To satisfy the zero torsion conditions (8), see equations in footnote 15 , let us consider a special class of generating functions and sources when, for instance, $\bar{\Psi}(\tau)=\overline{\check{\Psi}}\left(\tau, x^{i}, t\right)$, when $\left(\partial_{i} \overline{\check{\Psi}}\right)^{*}=\partial_{i}\left(\check{\check{\Psi}}^{*}\right)$ and $\bar{¥}\left(\tau, x^{i}, t\right)=\bar{¥}[\bar{\Psi}]=\bar{¥}(\tau)$, or $\bar{¥}=$ const. For such classes of entropic quasiperiodic generating functions and sources, the nonlinear symmetries (35) are written $\bar{\Lambda}(\tau) \check{\check{\Psi}}^{2}=$ $\check{\check{\Phi}}^{2}|\check{\Psi}|-\int d t \check{\check{\Phi}}^{2}|\check{\Psi}|^{*}, \check{\Phi}^{2}=-4 \bar{\Lambda}(\tau) \check{h}_{3}(\tau, r, \theta, t), \check{\check{\Psi}}^{2}=$ $\int d t \bar{¥}(\tau, r, \theta, t) \overline{\breve{h}}_{3}^{*}(\tau, r, \theta, t)$. Using these formulas, we conclude that the coefficient $\bar{h}_{4}(\tau)=\bar{h}_{4}\left(\tau, x^{i}, t\right)$ can be considered also as generating function for entropic cosmological solutions. For such LC-configurations, there are some parametric on $\tau$ functions $\overline{\check{A}}\left(\tau, x^{i}, t\right)$ and $n\left(\tau, x^{i}\right)$ when the Nconnection coefficients are computed

$$
\begin{aligned}
& \bar{n}_{k}(\tau)=\overline{\breve{n}}_{k}(\tau)=\partial_{k} \bar{n}\left(\tau, x^{i}\right) \text { and }
\end{aligned}
$$

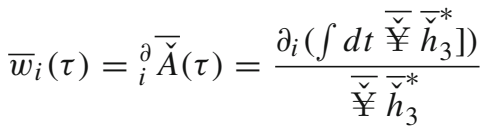

$$
\begin{aligned}
& =\frac{\partial_{i} \check{\Psi}}{\check{\Psi}^{*}}=\frac{\partial_{i}\left[\int d t \check{\dddot{\Psi}}\left(\check{\Phi}^{2}\right)^{*}\right]}{\check{\Psi}\left(\check{\Phi}^{2}\right)^{*}} .
\end{aligned}
$$

Summarizing above formulas, we construct new classes of locally anisotropic cosmological solutions as ub GR defined as subclasses of solutions (39) with zero torsion but with entropic quasiperiodic geometric flow evolution, cients are not zero for $N_{k}^{3}\left(\tau=\partial_{k} \bar{n}\right.$ and $N_{i}^{4}\left(\tau=\partial_{i} \overline{\check{A}}\right.$. They encode entropic quasiperiodic structures. We can analyze certain nonholonomic cosmological configurations determined, for instance, by data $\left(\check{\Psi}, \bar{\Psi}, h_{3}^{[0]}, \overline{\check{n}}_{k}\right)$, when $\partial_{k} \bar{n} \rightarrow$ 0 and $\bar{w}_{i}=\partial_{i} \check{A} \rightarrow 0$. Zero values can be fixed also by certain additional nonholonomic constraints. Choosing data $\left(\bar{\Psi}(\tau, t), \bar{\Psi}(\tau, t), h_{3}^{[0]}=\right.$ const,$\overline{\breve{n}}_{k}=$ const $)$, we can generate (off-) diagonal entropic metrics of Bianchi, or FLRW, types and generalizations to other type configurations $g_{\alpha \beta}(\tau, t)$ in GR modified under geometric flow evolution.

\section{Entropic quasiperiodic flows and cosmological solutions}

The goal of this section is to consider physical implications of models with entropic and quasiperiodic flow evolution of locally anisotropic and inhomogeneous cosmological spacetimes.

\subsection{The AFDM for entropic flow cosmological solutions}

We outline the key steps on the AFDM for generating cosmological solutions with geometric flows and Killing symmetry on $\partial_{3}$. Considering a nonholonomic deformation procedure for a generating function $g_{3}(\tau)=\bar{h}_{3}\left(\tau, x^{i}, y^{3}\right)(36)$, cosmological constants $\bar{\Lambda}(\tau)$ and sources ${ }_{h} \bar{¥}(\tau)={ }_{h} \bar{\Psi}\left(\tau, x^{k}\right)$ and $\bar{¥}(\tau)=\bar{¥}\left(\tau, x^{k}, t\right)$, see parameterizations (26) and nonlinear symmetries (35), we construct exact solutions of the system of nonlinear PDEs for emergent cosmology (34).

Typical cosmological solutions of this class are parameterised

$$
\begin{aligned}
& d s^{2}=e^{\psi\left(\tau, x^{k}\right)}\left[\left(d x^{1}\right)^{2}+\left(d x^{2}\right)^{2}\right]
\end{aligned}
$$

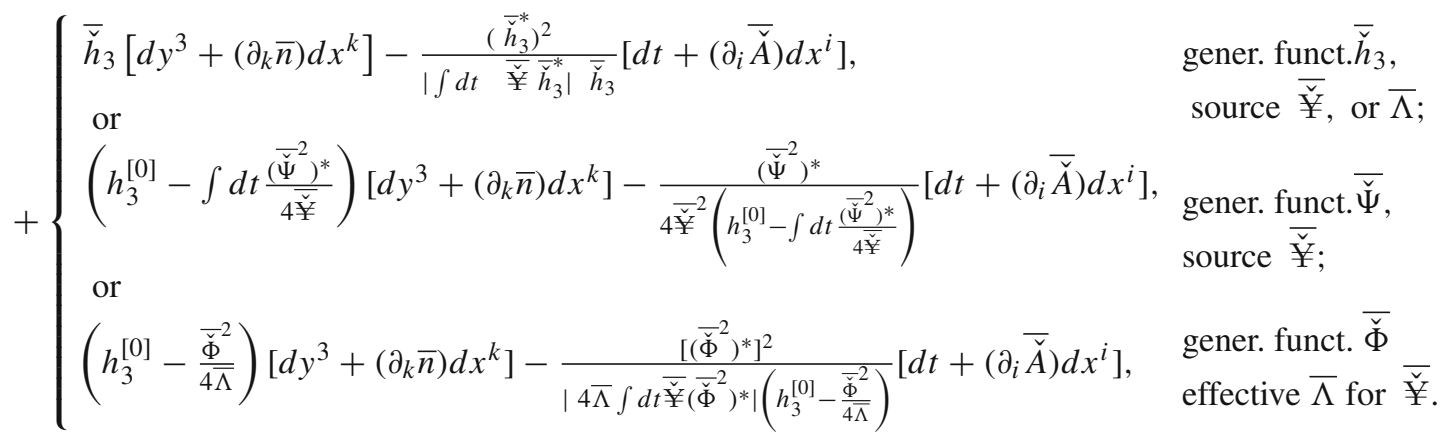

Such cosmological metrics are generic off-diagonal and define new classes of solutions if the anholonomy coeffi- 


$$
\begin{aligned}
d s^{2} & =e^{\psi\left(\tau, x^{k}\right)}\left[\left(d x^{1}\right)^{2}+\left(d x^{2}\right)^{2}\right]+\bar{h}_{3}(\tau) \\
& \times\left[d y^{3}+\left({ }_{1} n_{k}+4_{2} n_{k} \int d t \frac{\left(\bar{h}_{3}^{*}(\tau)\right)^{2}}{\left|\int d t \bar{\Psi}(\tau) \bar{h}_{3}^{*}\right|\left(\bar{h}_{3}(\tau)\right)^{5 / 2}}\right) d x^{k}\right] \\
& -\frac{\left[\bar{h}_{3}^{*}(\tau)\right]^{2}}{\left|\int d t \bar{\Psi}(\tau)\left(h_{4} \diamond(\tau)\right)\right| h_{4}}\left[d t+\frac{\partial_{i}\left(\int d t \bar{\Psi}(\tau) \bar{h}_{3}^{*}(\tau)\right)}{\bar{\Psi}(\tau) \bar{h}_{3}^{*}(\tau)} d x^{i}\right] .
\end{aligned}
$$

Such quadratic line elements are time dual to the stationary ones which can be obtained via transforms (33).

\subsection{Nonlinear PDEs for entropic quasiperiodic cosmology}

We analyse two possibilities to transform the entropic flow modified Einstein equations (23) into systems of nonlinear PDEs (29)-(32) with generic off-diagonal or diagonal solutions depending in explicit form on a evolution parameter, a time like variable and two space like coordinates. In the first case, there are considered entropic quasiperiodic sources determined by some additive or general nonlinear functionals for effective matter fields. In the second case, respective nonlinear functionals determining quasiperiodic solutions for entropic configurations are prescribed for generating functions subjected to nonlinear symmetries (35). We also note that is possible to construct certain classes of locally anisotropic and inhomogeneous cosmological solutions using nonlinear/additive functionals both for generating functions and (effective) sources.

\subsubsection{Cosmological solutions for entropic quasiperiodic sources}

Cosmological configurations generated by additive entropic functionals for sources: For this class of cosmological solutions, we consider an additive functional for an entropic quasiperiodic source of type $\bar{¥}\left(\tau, x^{i}, t\right)(26)$,

$$
\begin{aligned}
\text { as } \bar{¥}(\tau)= & { }^{a s} \bar{¥}\left(\tau, x^{i}, t\right)={ }^{f l} \bar{¥}\left(\tau, x^{i}, t\right) \\
& +{ }^{m} \bar{¥}\left(\tau, x^{i}, t\right)+{ }^{F} \bar{¥}\left(\tau, x^{i}, t\right) \\
& +{ }_{0}^{i n t} \bar{¥}\left(\tau, x^{i}, t\right)+{ }_{0}^{\chi} \bar{¥}\left(\tau, x^{i}, t\right) .
\end{aligned}
$$

There is also an associated additive cosmological constant as $\bar{\Lambda}(\tau)(40)$ related to different types of generating functions via nonlinear symmetries (35) when the Eq. (30) transforms into $\bar{\varpi}^{*} \bar{h}_{3}^{*}=2 \bar{h}_{3} \bar{h}_{4}$ as $\bar{¥}(\tau)$ and can be integrated on time like variable $y^{4}=t$. The systems of nonlinear PDEs (34) can be integrated following the AFDM explained in details in $[55-60,63,64]$ (see sections on exact and parametric cosmological solutions in MGTs) and [33-41], for solutions with nonholonomic cosmological Ricci flows, and citations therein. Such generic off-diagonal cosmological solutions are parameterized in the form

$d s^{2}=e^{\psi\left(\tau, x^{k}\right)}\left[\left(d x^{1}\right)^{2}+\left(d x^{2}\right)^{2}\right]+\bar{h}_{3}(\tau)$

$$
\begin{aligned}
& \times\left[d y^{3}+\left({ }_{1} n_{k}\left(\tau, x^{i}\right)+4_{2} n_{k}\left(\tau, x^{i}\right)\right.\right. \\
& \left.\left.\times \int d t \frac{\left[\bar{h}_{3} *(\tau)\right]^{2}}{\left|\int d t^{a s} \bar{¥}(\tau) \bar{h}_{3}^{*}(\tau)\right|\left[\bar{h}_{3}(\tau)\right]^{5 / 2}}\right) d x^{k}\right] \\
& -\frac{\left[\bar{h}_{3}^{*}(\tau)\right]^{2}}{\left|\int d t^{a s} \bar{¥}(\tau) \bar{h}_{3}^{*}(\tau)\right| \bar{h}_{3}(\tau)} \\
& \times\left[d t+\frac{\partial_{i}\left(\int d t^{a s} \bar{¥}(\tau) \bar{h}_{3} *(\tau)\right)}{a s \bar{¥}(\tau) \bar{h}_{3}^{*}(\tau)} d x^{i}\right] .
\end{aligned}
$$

In such quadratic linear elements, we have to fix a sign of the coefficient $\bar{h}_{3}\left(\tau, x^{k}, t\right)$ which describes relativistic flow evolution with a generating function with Killing symmetry on $\partial_{3}$ determined by sources $\left(h \bar{¥}(\tau),{ }^{a s} \bar{¥}(\tau)\right)$. Such entropic and quasiperiodic flow solutions are of type (39) and can be re-written equivalently with coefficients stated as functionals of ${ }^{a s} \bar{\Phi}\left(\tau, x^{i}, t\right)$ and ${ }^{a s} \bar{\Psi}\left(\tau, x^{i}, t\right)$.

We can extract from off-diagonal d-metrics (42) certain cosmological LC-configurations determined by entropic quasiperiodic sources by imposing additional zero torsion constraints. Such anholonomy conditions restrict the respective classes of generating functions $\left(\bar{h}_{3}\left(\tau, x^{i}, t\right), \overline{\breve{\Psi}}\left(\tau, x^{i}, t\right)\right.$ and/or $\left.\bar{\Phi}\left(\tau, x^{i}, t\right)\right)$ for $\left.\bar{n}\left(\tau, x^{i}\right), \bar{A}\left(\tau, x^{i}, t\right)\right)$ and sources as $\bar{¥}(\tau)(41)$ and ${ }^{a s} \bar{\Lambda}(\tau)(40)$,

$$
\begin{gathered}
d s^{2}=e^{\psi(\tau)}\left[\left(d x^{1}\right)^{2}+\left(d x^{2}\right)^{2}\right]+\overline{\breve{h}}_{3}(\tau)\left[d y^{3}+\left(\partial_{k} \bar{n}(\tau)\right) d x^{k}\right] \\
-\frac{\left[\overline{\breve{h}}_{3}^{*}(\tau)\right]^{2}}{\left|\int d t^{a s} \overline{\check{Y}}(\tau) \overline{\breve{h}}_{3} *(\tau)\right| \bar{h}_{3}(\tau)}\left[d t+\left(\partial_{i} \overline{\check{A}}(\tau)\right) d x^{i}\right] .
\end{gathered}
$$

The d-metrics (42) and/or (43) define off-diagonal cosmological solutions generated by entropic quasiperiodic additive sources ${ }^{a s} \bar{¥}(\tau)$ and/or ${ }^{a s} \bar{¥}(\tau)$. The terms (41) encode and model respectively contributions of standard and/or dark matter fields and effective entropic evolution sources. Such values can be can be prescribed in certain forms being compatible to observational data of cosmological (and geometric/entropic) evolution for dark matter distributions with possible quasiperiodic, aperiodic, pattern forming, solitonic nonlinear wave interactions.

Cosmological solutions for nonlinear entropic quasiperiodic functionals for sources: Such classes of exact cosmological solutions can be generated by nonlinear quasiperiodic functionals for effective sources, ${ }^{q} \bar{\Psi}(\tau)=q p \bar{¥}\left(\tau, x^{i}, t\right)=$ $q p \bar{¥}\left[{ }^{f l} \bar{¥},{ }^{m} \bar{¥},{ }^{F} \bar{¥},{ }_{0}^{i n t} \bar{¥}+{ }_{0}^{\chi} \bar{¥}\right]$, see functional dependencies in (41), subjected to nonlinear symmetries (35). Applying the AFDM, we construct entropic flow cosmological solutions of with nonlinear sources,

$d s^{2}=e^{\psi(\tau)}\left[\left(d x^{1}\right)^{2}+\left(d x^{2}\right)^{2}\right]+\bar{h}_{3}(\tau)$ 


$$
\begin{aligned}
& \times\left[d y^{3}+\left({ }_{1} n_{k}(\tau)+4_{2} n_{k}(\tau)\right.\right. \\
& \left.\left.\times \int d t \frac{\left[\bar{h}_{3}^{*}(\tau)\right]^{2}}{\left|\int d t^{q p} \bar{\Psi}(\tau) \bar{h}_{3} *(\tau)\right|\left[\bar{h}_{3}(\tau)\right]^{5 / 2}}\right) d x^{k}\right] \\
& -\frac{\left[\bar{h}_{3}{ }^{*}(\tau)\right]^{2}}{\left|\int d t^{q p} \bar{¥}(\tau) \bar{h}_{3}{ }^{*}(\tau)\right| \bar{h}_{3}(\tau)} \\
& \times\left[d t+\frac{\left.\partial_{i}\left(\int d t^{q p} \bar{\Psi}(\tau) \bar{h}_{3}^{*}(\tau)\right]\right)}{q p \bar{\Psi}(\tau) \bar{h}_{3}{ }^{*}(\tau)} d x^{i}\right] .
\end{aligned}
$$

For LC-configurations, we obtain

$$
\begin{gathered}
d s^{2}=e^{\psi(\tau)}\left[\left(d x^{1}\right)^{2}+\left(d x^{2}\right)^{2}\right]+\overline{\breve{h}}_{3}(\tau)\left[d y^{3}+\left(\partial_{k} \bar{n}(\tau)\right) d x^{k}\right] \\
-\frac{\left[\overline{\breve{h}}_{3}^{*}(\tau)\right]^{2}}{\left|\int d t q p \overline{\check{Y}}^{2}(\tau) \bar{h}_{3} *(\tau)\right| \overline{\breve{h}}_{3}(\tau)}\left[d t+\left(\partial_{i} \overline{\breve{A}}(\tau)\right) d x^{i}\right] .
\end{gathered}
$$

For additive functionals for cosmological entropic and quasiperiodic sources, the formulas (44) and (45) transforms respectively into quadratic linear elements (42) and (43). Fixing a value $\tau_{0}$, we obtain cosmological solutions for Ricci solitons and MGTs.

\subsubsection{Cosmological configurations with nonstationary entropic generating functions}

In this section, the sources for (effective) matter fields and geometric flows are defined by arbitrary functions $\bar{¥}_{v}^{\mu}(\tau)=$ $\left[h \bar{¥}\left(\tau, x^{k}\right), \bar{¥}\left(\tau, x^{k}, t\right)\right]$. The quasiperiodic structure will be stated for some additive or general nonlinear functionals for the generating functions.

Cosmological metrics with additive entropic generating functions: Such functionals are

$$
\begin{aligned}
a \bar{\Phi}(\tau)= & { }^{a} \bar{\Phi}\left(\tau, x^{i}, t\right)={ }^{f l} \bar{\Phi}\left(\tau, x^{i}, t\right) \\
& +{ }^{m} \bar{\Phi}\left(\tau, x^{i}, t\right)+{ }^{F} \bar{\Phi}\left(\tau, x^{i}, t\right) \\
& +{ }_{0}^{i n t} \bar{\Phi}\left(\tau, x^{i}, t\right)+{ }_{0}^{\chi} \bar{\Phi}\left(\tau, x^{i}, t\right) .
\end{aligned}
$$

The values ${ }_{0}^{\text {int }} \bar{\Phi}(\tau)={ }_{0}^{\text {int }} \bar{\Phi}\left(\tau, x^{i}, t\right)={ }^{i n t} \Phi[\tau, \varsigma, \bar{b}]$ and ${ }_{0}^{\chi} \bar{\Phi}(\tau)={ }_{0}^{\chi} \bar{\Phi}\left(\tau, x^{i}, t\right)=\chi \bar{\Phi}[\tau, \varsigma, \bar{b}]$ are functionals on certain quasiperiodic (space time like QC or other type aperiodic, solitonic structures) given by functions $\varsigma$ and/or $\bar{b}$ subjected to conditions of type (12) and/or (13). The terms in the sum (46) correspond to the Lagrange densities (14) and energy-momentum tensors (15) nonholonomically parameterised by sources (26). Changing systems of references and coordinates, we can compute sums of functionals for sources of type (41) using nonlinear symmetries (35) considering an associated additive cosmological constant ${ }^{a s} \bar{\Lambda}(\tau)$ (40). For ${ }^{a} \bar{\Phi}(\tau)$ (46), the Eq. (30) transforms into a functional equation $\bar{\varpi}^{*}(\tau)\left[{ }^{a} \bar{\Phi}(\tau), \bar{\Lambda}(\tau)\right] \bar{h}_{3}^{*}(\tau)\left[{ }^{a} \bar{\Phi}(\tau), \bar{\Lambda}(\tau)\right]=$ $2 \bar{h}_{3}(\tau)\left[{ }^{a} \bar{\Phi}(\tau), \bar{\Lambda}(\tau)\right] \bar{h}_{4}(\tau)\left[{ }^{a} \bar{\Phi}(\tau), \bar{\Lambda}(\tau)\right] \bar{¥}(\tau)$, and related analogs of (31) and (32) in the decoupled system of nonlinear PDEs (34). Such equations and their solutions can be written equivalently in different forms with additive functionals of type ${ }^{a} \bar{h}_{3,4}$ and/or ${ }^{a} \bar{\Psi}$ and respective nonlinear functionals for the coefficients in respective equations. Applying the AFDM as in the Sect. 4.3.1, we generate a class of parametric solutions of (34) parameterized in a form similarly to (39)

$$
\begin{aligned}
d s^{2}= & e^{\psi\left(\tau, x^{k}\right)}\left[\left(d x^{1}\right)^{2}+\left(d x^{2}\right)^{2}\right]+\left[h_{3}^{[0]}\left(\tau, x^{i}\right)-\frac{a \bar{\Phi}^{2}(\tau)}{4 \bar{\Lambda}(\tau)}\right] \\
& \times\left[d y^{3}+\left({ }_{1} n_{k}\left(\tau, x^{i}\right)+{ }_{2} n_{k}\left(\tau, x^{i}\right)\right.\right. \\
& \left.\left.\times \int d t \frac{\left.\left[{ }^{a} \bar{\Phi}^{2}\right)^{*}(\tau)\right]^{2}}{4\left|\bar{\Lambda}(\tau) \int d t \bar{\Psi}(\tau)\left({ }^{a} \bar{\Phi}^{2}(\tau)\right)^{*}\right|\left(h_{3}^{[0]}\left(\tau, x^{i}\right)-\frac{a \bar{\Phi}^{2}(\tau)}{4 \bar{\Lambda}(\tau)}\right)^{5 / 2}}\right) d x^{k}\right] \\
& -\frac{\left.\left[{ }^{a} \bar{\Phi}^{2}\right)^{*}(\tau)\right]^{2}}{4\left|\bar{\Lambda}(\tau) \int d t \bar{\Psi}(\tau)\left({ }^{a} \bar{\Phi}^{2}\right)^{*}(\tau)\right|\left[h_{3}^{[0]}\left(\tau, x^{i}\right)-\frac{a \bar{\Phi}^{2}(\tau)}{4 \bar{\Lambda}(\tau)}\right]} \\
& \times\left[d t+\frac{\partial_{i}\left[\int d t \bar{\Psi}(\tau)\left({ }^{a} \bar{\Phi}^{2}\right)^{*}(\tau)\right]}{\bar{\Psi}(\tau)\left({ }^{a} \bar{\Phi}^{2}\right)^{*}(\tau)} d x^{i}\right],
\end{aligned}
$$

for integration functions $h_{3}^{[0]}\left(\tau, x^{i}\right),{ }_{1} n_{k}\left(\tau, x^{i}\right)$ and ${ }_{2} n_{k}\left(\tau, x^{i}\right)$.

LC-configurations can be extracted from (47) imposing additional integrability conditions on coefficients resulting in zero nonholonomic torsion. Such solutions can be considered both for relativistic entropic Ricci solitons and in GR, being parameterized as in (40),

$$
\begin{aligned}
d s^{2}= & e^{\psi\left(\tau, x^{k}\right)}\left[\left(d x^{1}\right)^{2}+\left(d x^{2}\right)^{2}\right] \\
& +\left[h_{3}^{[0]}\left(\tau, x^{i}\right)-\frac{\check{\Phi}^{2}(\tau)}{4 \bar{\Lambda}(\tau)}\right]\left[d y^{3}+\left(\partial_{k} \bar{n}\left(\tau, x^{i}\right)\right) d x^{k}\right] \\
& -\frac{\left[\left({ }^{a} \bar{\Phi}^{2}\right)^{*}(\tau)\right]^{2}}{4\left|\bar{\Lambda}(\tau) \int d t \overline{\check{\Psi}}(\tau)\left(a \check{\Phi}^{2}\right)^{*}(\tau)\right|\left(h_{3}^{[0]}\left(\tau, x^{i}\right)-\frac{\overline{\check{\Phi}}^{2}(\tau)}{4 \bar{\Lambda}(\tau)}\right)} \\
& \times\left[d t+\left(\partial_{i} \overline{\check{A}}\left(\tau, x^{k}, t\right)\right) d x^{i}\right] .
\end{aligned}
$$

We can consider small parametric decompositions and frame/coordinate transforms (in a more general context, we can elaborate a formalism of $\eta$ - and $\varepsilon$-polarization functions as for BH solutions but with time like dependence for cosmological configurations) in order to relate new classes of solutions (47) and/or (48) to some well known (off-) diagonal cosmological metrics.

Cosmological configurations with nonlinear entropic functionals for generating functions: Instead of additive generating functionals ${ }^{a} \bar{\Phi}(\tau)$ (46), we can work with nonlinear quasiperiodic generating functionals ${ }^{q p} \bar{\Phi}(\tau)={ }^{q p} \bar{\Phi}\left(\tau, x^{i}, t\right)$ $={ }^{q p} \bar{\Phi}\left[{ }^{f l} \bar{\Phi},{ }^{m} \bar{\Phi},{ }^{F} \bar{\Phi},{ }_{0}^{i n t} \bar{\Phi},{ }_{0}^{\chi} \bar{\Phi}\right]$ characterized by nonlinear symmetries of type (35). The Eq. (30) transforms into a 
nonlinear functional equation, $\bar{\varpi}^{*}(\tau)\left[{ }^{q p} \bar{\Phi}(\tau), \bar{\Lambda}(\tau)\right] \bar{h}_{3}^{*}(\tau)$ $\left.{ }^{q p} \bar{\Phi}(\tau), \bar{\Lambda}(\tau)\right]=2 \bar{h}_{3}(\tau)\left[{ }^{q p} \bar{\Phi}(\tau), \bar{\Lambda}(\tau)\right] \bar{h}_{4}(\tau)\left[{ }^{q p} \bar{\Phi}(\tau)\right.$, $\bar{\Lambda}(\tau)] \bar{¥}(\tau)$, which can be solved together with other equations with decoupling (34). We obtain such solutions:

$$
\begin{aligned}
d s^{2}= & e^{\psi\left(\tau, x^{k}\right)}\left[\left(d x^{1}\right)^{2}+\left(d x^{2}\right)^{2}\right]+\left[h_{3}^{[0]}\left(\tau, x^{i}\right)-\frac{q p \bar{\Phi}^{2}(\tau)}{4 \bar{\Lambda}(\tau)}\right] \\
& \times\left[d y^{3}+\left({ }_{1} n_{k}\left(\tau, x^{i}\right)+{ }_{2} n_{k}\left(\tau, x^{i}\right)\right.\right. \\
& \left.\left.\times \int d t \frac{\left.\left[{ }^{q p} \bar{\Phi}^{2}\right)^{*}(\tau)\right]^{2}}{4\left|\bar{\Lambda}(\tau) \int d t \bar{¥}(\tau)\left(q p \bar{\Phi}^{2}\right)^{*}(\tau)\right|\left(h_{3}^{[0]}\left(\tau, x^{i}\right)-\frac{q p \bar{\Phi}^{2}(\tau)}{4 \bar{\Lambda}(\tau)}\right)^{5 / 2}}\right) d x^{k}\right] \\
& -\frac{\left.\left[{ }^{q p} \bar{\Phi}^{2}\right)^{*}(\tau)\right]^{2}}{4\left|\bar{\Lambda}(\tau) \int d t \bar{¥}(\tau)\left(q p \bar{\Phi}^{2}\right)^{*}(\tau)\right|\left(h_{3}^{[0]}\left(\tau, x^{i}\right)-\frac{{ }^{q p} \bar{\Phi}^{2}(\tau)}{4 \bar{\Lambda}(\tau)}\right)} \\
& \times\left[d t+\frac{\partial_{i}\left(\int d t \bar{¥}(\tau)\left({ }^{q p} \bar{\Phi}^{2}\right)^{*} \bar{¥}(\tau)\right)}{\bar{¥}(\tau)\left(q p \bar{\Phi}^{2}\right)^{*} \bar{¥}(\tau)} d x^{i}\right],
\end{aligned}
$$

where $h_{3}^{[0]}\left(\tau, x^{i}\right),{ }_{1} n_{k}\left(\tau, x^{i}\right)$ and ${ }_{2} n_{k}\left(\tau, x^{i}\right)$ are integration functions.

For zero torsion constraints, we extract LC-configurations,

$$
\begin{aligned}
d s^{2}= & e^{\psi\left(x^{k}\right)}\left[\left(d x^{1}\right)^{2}+\left(d x^{2}\right)^{2}\right] \\
& +\left(h_{3}^{[0]}\left(\tau, x^{i}\right)-\frac{q p \bar{\Phi}^{2}(\tau)}{4 \bar{\Lambda}(\tau)}\right) \\
& \times\left[d y^{3}+\left(\partial_{k} \bar{n}\left(\tau, x^{i}\right)\right) d x^{k}\right] \\
& -\frac{\left[\left({ }^{2} p \overline{\check{\Phi}}^{2}\right)^{*}(\tau)\right]^{2}}{4\left|\bar{\Lambda}(\tau) \int d t \bar{\Psi}(\tau)\left(q p \check{\Phi}^{2}\right)^{*}(\tau)\right|\left(h_{3}^{[0]}\left(\tau, x^{i}\right)-\frac{q p \overline{\check{\Phi}}^{2}(\tau)}{4 \bar{\Lambda}(\tau)}\right)} \\
& \times\left[d t+\left(\partial_{i} \overline{\check{A}}\left(\tau, x^{i}, t\right)\right) d x^{i}\right] .
\end{aligned}
$$

The coefficients of d-metrics (49) and (50) can be chosen to be of necessary smooth class and involve certain entropic, quasiperiodic, stochastic sources and fractional derivative processes. Such nonholonomic deformation and generalized transforms can be constructing with changing the topological spacetime structure and modeling certain dark energy and dark matter effects as results of entropic quasiperiodic flows or nonholonomic deformations of certain prime cosmological solutions.

\subsubsection{Emergent quasiperiodic cosmology from both generating functionals and sources}

We can generate entropic flow cosmological solutions using generalized quasiperiodic nonlinear functionals both for generating functions, ${ }^{q p} \bar{\Phi}(\tau)$, and nonlinear functionals for (effective) sources, ${ }^{q p} ¥(\tau)$, see above formulas. Such data are connected via nonlinear symmetries of type (35), when ${ }^{q p} \bar{\Lambda}(\tau)^{q p} \bar{\Psi}^{2}(\tau)=\left.\left.{ }^{q p} \bar{\Phi}^{2}(\tau)\right|^{q p} \bar{¥}(\tau)\left|-\int d t^{q p} \bar{\Phi}^{2}(\tau)\right|^{q p} \bar{¥}(\tau)\right|^{*}$.
Similar nonlinear symmetries exist for additive functionals both for the gravitational fields and (effective) sources, with (46) and (41) and can be written in a particular form ${ }^{a} \bar{\Lambda}(\tau)^{a} \bar{\Psi}^{2}(\tau)=\left.\left.{ }^{a} \bar{\Phi}^{2}\right|^{a} \bar{¥}(\tau)\left|-\int d t^{a} \bar{\Phi}^{2}\right|^{a} \bar{¥}(\tau)\right|^{*}$. Applying the AFDM (using data ( ${ }^{q p} \bar{\Psi}(\tau),{ }^{q p} \bar{¥}(\tau)$ ), and/or, equivalently, $\left.\left({ }^{q p} \bar{\Phi}(\tau),{ }^{q p} \bar{\Lambda}(\tau)\right)\right)$, we construct multi-functional nonlinear entropic quasiperiodic cosmological configurations,

$$
\begin{aligned}
& d s^{2}=e^{\psi\left(\tau, x^{k}\right)}\left[\left(d x^{1}\right)^{2}+\left(d x^{2}\right)^{2}\right]+\left(h_{3}^{[0]}\left(\tau, x^{i}\right)-\frac{q p \bar{\Phi}^{2}(\tau)}{4 q p \bar{\Lambda}(\tau)}\right) \\
& \times\left[d y^{3}+\left({ }_{1} n_{k}\left(\tau, x^{i}\right)+{ }_{2} n_{k}\left(\tau, x^{i}\right)\right.\right. \\
& \left.\left.\times \int d t \frac{\left[\left({ }^{q p} \bar{\Phi}^{2}\right)^{*}(\tau)\right]^{2}}{4^{q p}\left|\bar{\Lambda}(\tau) \int d t^{q p} \bar{¥}\left(q p \bar{\Phi}^{2}\right)^{*}\right|\left(h_{3}^{[0]}\left(\tau, x^{i}\right)-\frac{q p \bar{\Phi}^{2}(\tau)}{4 q p \bar{\Lambda}(\tau)}\right)^{5 / 2}}\right) d x^{k}\right] \\
& -\frac{\left.\left[{ }^{q p} \bar{\Phi}^{2}\right)^{*}(\tau)\right]^{2}}{\left.4\right|^{q p} \bar{\Lambda}(\tau) \int d t^{q p} \bar{¥}(\tau)\left({ }^{q p} \bar{\Phi}^{2}\right)^{*} \mid\left(h_{3}^{[0]}\left(\tau, x^{i}\right)-\frac{q p \bar{\Phi}^{2}(\tau)}{4 q p \bar{\Lambda}(\tau)}\right)} \\
& \times\left[d t+\frac{\left.\partial_{i}\left(\int d t t^{q p} \bar{¥}(\tau)\left({ }^{q p} \bar{\Phi}^{2}\right)^{*}(\tau)\right]\right)}{q p \bar{¥}(\tau)\left(q p \bar{\Phi}^{2}\right)^{*}(\tau)} d x^{i}\right],
\end{aligned}
$$

where for integration functions $h_{3}^{[0]}\left(\tau, x^{i}\right),{ }_{1} n_{k}\left(\tau, x^{i}\right)$ and ${ }_{2} n_{k}\left(\tau, x^{i}\right)$.

For LC-configurations, we obtain multi-functional nonlinear generalizations of (49) and (50) modelling locally anisotropic and inhomogeneous solutions in entropic flow gravity and GR,

$$
\begin{aligned}
d s^{2}= & e^{\psi\left(\tau, x^{k}\right)}\left[\left(d x^{1}\right)^{2}+\left(d x^{2}\right)^{2}\right]+\left(h_{3}^{[0]}\left(\tau, x^{i}\right)-\frac{q p \check{\Phi}^{2}(\tau)}{4 q p \bar{\Lambda}(\tau)}\right) \\
& \times\left[d y^{3}+\left(\partial_{k} \bar{n}\left(\tau, x^{i}, t\right)\right) d x^{k}\right] \\
& -\frac{\left[\left({ }^{q p} \bar{\Phi}^{2}\right)^{*}(\tau)\right]^{2}}{\left.4\right|^{q p} \bar{\Lambda}(\tau) \int d t^{q p} \overline{\check{\Psi}}(\tau)\left(q p \check{\Phi}^{2}\right)^{*}(\tau) \mid\left(h_{3}^{[0]}\left(\tau, x^{i}\right)-\frac{q p \bar{\Phi}^{2}(\tau)}{4 q p \bar{\Lambda}(\tau)}\right)} \\
& \times\left[d t+\left(\partial_{i} \overline{\check{A}}\left(\tau, x^{i}, t\right)\right) d x^{i}\right] .
\end{aligned}
$$

The classes of cosmological solutions (51) and (52) describe off-diagonal entropic non-stationary configurations determined by multi-functional nonlinear quasiperiodic structures.

\subsection{Cosmological metrics evolving in entropic quasiperiodic media}

Generic off-diagonal entropic and quasiperiodic cosmological solutions are constructed in terms of $\eta$-polarization functions following the AFDM method for parametric $\tau$ and time like depending evolution. We consider a primary cosmological d-metric $\stackrel{\mathrm{g}}{\mathrm{g}}(28)$ defined by data $\left[\stackrel{\circ}{g}_{i}\left(x^{k}, t\right), \stackrel{\circ}{g}_{a}=\right.$ $\left.\grave{h}_{a}\left(x^{k}, t\right) ; \stackrel{\circ}{N}_{k}^{3}=\stackrel{\circ}{n}_{k}\left(x^{i}, t\right), \stackrel{\circ}{N}_{k}^{4}=\stackrel{\circ}{w}_{k}\left(x^{i}, t\right)\right]$ which can be diagonalized for a FLRW cosmological metric by frame/ 
coordinate transforms. ${ }^{16}$ The cosmological entropic quasiperiodic y target metrics $\mathbf{\mathbf { g }}(\tau)$ of type (27) are generated by nonholonomic deformations

$$
\begin{aligned}
\stackrel{\circ}{\mathbf{g}} & \rightarrow \overline{\mathbf{g}}(\tau)=\left[\bar{g}_{i}\left(\tau, x^{k}\right)=\bar{\eta}_{i}\left(\tau, x^{k}, t\right) \stackrel{\circ}{g}_{i}, \bar{g}_{b}\left(\tau, x^{k}, t\right)\right. \\
& \left.=\bar{\eta}_{b}\left(\tau, x^{k}, t\right) \stackrel{\circ}{g}_{b}, \bar{N}_{i}^{a}\left(\tau, x^{k}, t\right)=\bar{\eta}_{i}^{a}\left(\tau, x^{k}, t\right) \stackrel{\circ}{N}_{i}^{a}\right],
\end{aligned}
$$

when overlined symbols are used for distinguishing cosmological d-metrics from stationary ones studied in previous sections. The quadratic line elements corresponding to target locally anisotropic and inhomogeneous cosmological metrics $\overline{\mathbf{g}}(\tau)=\overline{\mathbf{g}}\left(\tau, x^{k}, t\right)$ can be written in terms of gravitational polarization functions,

$$
\begin{aligned}
d s^{2}= & \bar{\eta}_{1}\left(\tau, x^{i}, t\right) \stackrel{\circ}{g}_{1}\left(x^{i}, t\right)\left[d x^{1}\right]^{2} \\
& +\bar{\eta}_{2}\left(\tau, x^{i}, t\right) \stackrel{\circ}{g}_{2}\left(x^{i}, t\right)\left[d x^{1}\right]^{2} \\
& +\bar{\eta}_{3}\left(\tau, x^{i}, t\right) \stackrel{\circ}{h}_{3}\left(x^{i}, t\right) \\
& \times\left[d y^{3}+\bar{\eta}_{i}^{3}\left(\tau, x^{i}, t\right) \stackrel{\circ}{N}_{i}^{3}\left(x^{k}, t\right) d x^{i}\right]^{2} \\
& +\bar{\eta}_{4}\left(\tau, x^{i}, t\right) \stackrel{\circ}{h}_{4}\left(x^{i}, t\right) \\
& \times\left[d t+\bar{\eta}_{i}^{4}\left(\tau, x^{k}, t\right) \stackrel{\circ}{N}_{i}^{4}\left(x^{k}, t\right) d x^{i}\right]^{2} .
\end{aligned}
$$

The $\eta$-coefficients will be constructed in some explicit forms determined by entropic quasiperiodics generation functions and/or effective sources as solutions of the system of nonlinear PDEs (34).

\subsubsection{Cosmological evolutions generated by nonstationary entropic sources}

We consider entropic sources of type $q p \bar{¥}(\tau)=q p \bar{¥}\left(\tau, x^{i}, t\right)$ $=q p \bar{¥}\left[l \bar{¥},{ }^{m} \bar{¥}, F \bar{¥},{ }_{0}^{i n t} \bar{¥}+{ }_{0}^{\chi} \bar{¥}\right]$ as in (44) and (45) and compute the $\eta$-polarization functions following formulas

$$
\begin{aligned}
& \bar{\eta}_{i}(\tau)=e^{\psi\left(\tau, x^{i}\right)} / \stackrel{\circ}{g}_{i} ; \text { generating function } \\
& \bar{\eta}_{3}(\tau)=\bar{\eta}_{3}\left(\tau, x^{i}, t\right) ; \\
& \bar{\eta}_{4}(\tau)=-\frac{4\left[\left(\left|\bar{\eta}_{3}(\tau) \stackrel{\circ}{h}_{3}\right|^{1 / 2}\right)^{*}\right]^{2}}{\stackrel{\circ}{h}_{4}\left|\int d t^{q p} \bar{¥}(\tau)\left(\bar{\eta}_{3}(\tau) \stackrel{\circ}{h}_{3}\right)^{*}\right|} ; \\
& \bar{\eta}_{i}^{3}(\tau)=\frac{1 n_{k}\left(\tau, x^{i}\right)}{\stackrel{\circ}{n}_{k}}+4 \frac{2 n_{k}\left(\tau, x^{i}\right)}{\stackrel{\circ}{n}_{k}} \\
& \times \int d t \frac{\left(\left[\left(\bar{\eta}_{3}(\tau) \stackrel{\circ}{h}_{3}\right)^{-1 / 4}\right]^{*}\right)^{2}}{\left|\int d t q p \bar{¥}(\tau)\left(\bar{\eta}_{3}(\tau) \stackrel{\circ}{h}_{3}\right)^{*}\right|} ; \\
& \bar{\eta}_{k}^{4}(\tau)=\frac{\partial_{i} \int d t^{q p} \bar{¥}(\tau)\left(\bar{\eta}_{3}(\tau) \stackrel{\circ}{h}_{3}\right)^{*}}{{\overline{\dot{w}_{i}}}_{i} q p \bar{¥}(\tau)\left(\bar{\eta}_{3}(\tau) \stackrel{\circ}{h}_{3}\right)^{*}},
\end{aligned}
$$

where ${ }_{1} n_{k}\left(\tau, x^{i}\right)$ and ${ }_{2} n_{k}\left(\tau, x^{i}\right)$ are integration functions.

Using $\bar{\eta}_{3}\left(\tau, x^{i}, t\right)$ as a generating function, we can compute other types of generating functions of the same target cosmological d-metric subjected to nonlinear symmetries

\footnotetext{
16 In general, we can consider off-diagonal Bianchi anisotropic cosmological metrics or any cosmological solution in GR or MGTs.
}

(35),

$$
\begin{gathered}
\bar{\Phi}^{2}(\tau)=\left.4\right|^{q p} \bar{\Lambda}(\tau)\left[h_{3}^{[0]}\left(\tau, x^{k}\right)-\bar{\eta}_{3}\left(\tau, x^{i}, t\right) \stackrel{\circ}{h}_{3}\left(x^{k}, t\right)\right] \mid, \\
\left(\bar{\Psi}^{2}\right)^{*}(\tau)=-\int d t^{q p} \bar{¥}(\tau)\left[\bar{\eta}_{3}\left(\tau, x^{i}, t\right) \bar{\circ}_{3}\left(x^{i}, t\right)\right]^{*} .
\end{gathered}
$$

For integrable generating functionals and sources, when the constructions (15) are subjected to target off-diagonal cosmological metrics (40) with zero torsion, we obtain

$$
\begin{aligned}
& \bar{\eta}_{i}(\tau)=e^{\psi\left(\tau, x^{i}\right)} / \stackrel{\circ}{g}_{i} ; \bar{\eta}_{3}(\tau) \\
& =\overline{\grave{\eta}}_{3}\left(\tau, x^{i}, t\right) \text { as a generating function; } \\
& \bar{\eta}_{4}(\tau)=-\frac{4\left[\left(\left|\overline{\grave{\eta}}_{3}(\tau) \stackrel{\circ}{h}_{3}\right|^{1 / 2}\right)^{*}\right]^{2}}{\grave{h}_{4}\left|\int d t^{q p} \overline{\check{\varpi}}(\tau)\left(\overline{\check{\eta}}_{3} \stackrel{\circ}{h}_{3}\right)^{*}\right|} ; \\
& \bar{\eta}_{k}^{3}(\tau)=\frac{\partial_{k} \bar{n}\left(\tau, x^{i}\right)}{\stackrel{\circ}{n}_{k}} ; \bar{\eta}_{k}^{4}(\tau)=\frac{\partial_{k} \overline{\check{A}}\left(\tau, x^{i}, t\right)}{\stackrel{\stackrel{w}{w}}{k}} \text {. }
\end{aligned}
$$

In (53) and (54), the nonlinear functionals for the entropic quasiperiodic v-source and (effective) cosmological constant can be changed into additive functionals $q p \bar{¥}(\tau) \rightarrow$ as $\bar{¥}(\tau)$ and $q p \bar{\Lambda}(\tau) \rightarrow$ as $\bar{\Lambda}(\tau)$ which generates another classes of cosmological solutions.

\subsubsection{Cosmology from nonstationary entropic generating functions}

We can construct locally anisotropic and inhomogeneous cosmological solutions as nonholonomic deformations of some prime cosmological metrics when the coefficients of the d-metrics are determined nonlinear generating functionals ${ }^{q} p \bar{\Phi}(\tau)={ }^{q p} \bar{\Phi}\left(\tau, x^{i}, t\right)=q p \bar{\Phi}\left[l l \bar{\Phi}, m \bar{\Phi},{ }^{F} \bar{\Phi},{ }_{0}^{i n t} \bar{\Phi}, \underset{0}{\chi} \bar{\Phi}\right]$ as for (49). It is possible also to generate similar cosmological metrics by additive functionals $a \bar{\Phi}(\tau)$ (46) for prescribed families of effective sources $¥(\tau)=\bar{¥}\left(\tau, x^{i}, t\right)$ and cosmological constants $\bar{\Lambda}(\tau)$. Using formulas for nonlinear symmetries (35), we compute (recurrently) corresponding nonlinear functionals, ${ }^{q p} \bar{\eta}_{3}\left(\tau, x^{i}, t\right)$, or additive functionals, ${ }^{a} \bar{\eta}_{3}\left(\tau, x^{i}, t\right)$, and related polarization functions,

$$
\begin{gathered}
q p \bar{\Phi}^{2}(\tau)=4\left|\bar{\Lambda}(\tau)\left[h_{3}^{[0]}\left(\tau, x^{k}\right)-{ }^{q p} \bar{\eta}_{3}\left(\tau, x^{i}, t\right) \stackrel{\circ}{h}_{3}\left(x^{k}, t\right)\right]\right|, \\
\left({ }^{q p} \bar{\Psi}^{2}\right)^{*}(\tau)=-\int d t \bar{¥}(\tau)\left[{ }^{q p} \bar{\eta}_{3}\left(\tau, x^{i}, t\right) \bar{\circ}_{3}\left(x^{i}, t\right)\right]^{*} .
\end{gathered}
$$

The coefficients of quadratic elements of type (39) are recurrently computed,

$\bar{\eta}_{i}(\tau)=e^{\psi\left(\tau, x^{i}\right)} / \stackrel{\circ}{g}_{i} ;$

$\bar{\eta}_{3}(\tau)={ }^{q p} \bar{\eta}_{3}\left(\tau, x^{i}, t\right)$ as a generating function;

$\bar{\eta}_{4}(\tau)=-\frac{4\left[\left(\left.\left.\right|^{q p} \bar{\eta}_{3}(\tau) \stackrel{\circ}{h}_{3}\right|^{1 / 2}\right)^{*}\right]^{2}}{h_{4}\left|\int d t \bar{¥}(\tau)\left(q p \bar{\eta}_{3}(\tau) \stackrel{\circ}{h}_{3}\right)^{*}\right|} ;$

$\bar{\eta}_{i}^{3}(\tau)=\frac{1 n_{k}\left(\tau, x^{i}\right)}{\stackrel{\circ}{n}_{k}}+4 \frac{2 n_{k}\left(\tau, x^{i}\right)}{\stackrel{\circ}{n}_{k}}$ 


$$
\begin{gathered}
\times \int d t \frac{\left(\left[\left({ }^{q p} \bar{\eta}_{3}(\tau) \stackrel{\circ}{h}_{3}\right)^{-1 / 4}\right]^{*}\right)^{2}}{\left|\int d t \bar{\Psi}(\tau)\left({ }^{q p} \bar{\eta}_{3}(\tau) \stackrel{\circ}{h}_{3}\right)^{*}\right|} ; \\
\bar{\eta}_{k}^{4}(\tau)=\frac{\partial_{i} \int d t \bar{¥}(\tau)\left({ }^{q p} \bar{\eta}_{3}(\tau) \stackrel{\circ}{h}_{3}\right)^{*}}{\overline{\mathfrak{w}}_{i} \bar{\Psi}(\tau)\left({ }^{q p} \bar{\eta}_{3}(\tau) \stackrel{\circ}{h}_{3}\right)^{*}},
\end{gathered}
$$

where ${ }_{1} n_{k}\left(\tau, x^{i}\right)$ and ${ }_{2} n_{k}\left(\tau, x^{i}\right)$ are integration functions.

Target off-diagonal cosmological metrics (40) with zero torsion extracted from (55) can be generated by polarization functions

$\bar{\eta}_{i}(\tau)=e^{\psi\left(\tau, x^{i}\right)} / \stackrel{\circ}{g}_{i} ;$ generating function

$\bar{\eta}_{3}(\tau)=q p \overline{\check{\eta}}_{3}\left(\tau, x^{i}, t\right)$;

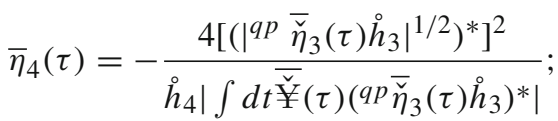

$\bar{\eta}_{k}^{3}(\tau)=\left(\partial_{k} \bar{n}\left(\tau, x^{i}\right)\right) / \stackrel{\circ}{n}_{k} ;$

$\bar{\eta}_{k}^{4}=\partial_{k} \overline{\check{A}}\left(\tau, x^{i}, t\right) / \stackrel{\circ}{w}_{k}$.

The cosmological solutions generated in this subsection describe entropic flow nonholonomic deformations of prime cosmological configurations (for instance, a FLRW, or Bianchi, type metric, and various modifications in accelerating cosmology) self-consistently imbedded into a quasiperiodic gravitational (dark energy) media.

\subsubsection{Cosmological configurations for entropic sources and generating functions}

We can construct more general classes of nonholonomic deformations of prime cosmological metrics generated by entropic quasiperiodic flow nonlinear quaisperiodic functionals both for the generating functions and (effective) sources. For such locally anisotropic and inhomogeneous cosmological models defined by nonlinear superpositions of cosmological solutions (53) and (55) when the coefficients of (44) are computed,

$\bar{\eta}_{i}(\tau)=e^{\psi\left(\tau, x^{i}\right)} / \stackrel{\circ}{g}_{i} ;$ generating function

${ }^{q p} \bar{\eta}_{3}(\tau)={ }^{q p} \bar{\eta}_{3}\left(\tau, x^{i}, t\right)$

$\bar{\eta}_{4}(\tau)=-\frac{4\left[\left(\left.\left.\right|^{q p} \bar{\eta}_{3}(\tau) \stackrel{\circ}{h}_{3}\right|^{1 / 2}\right)^{*}\right]^{2}}{\stackrel{\circ}{h}_{4}\left|\int d t^{q p} \bar{¥}(\tau)\left({ }^{q p} \bar{\eta}_{3}(\tau) \stackrel{\circ}{h}_{3}\right)^{*}\right|} ;$

$\bar{\eta}_{i}^{3}(\tau)=\frac{1 n_{k}(\tau)}{\stackrel{\circ}{n}_{k}}+\frac{4_{2} n_{k}(\tau)}{\stackrel{\circ}{n}_{k}}$

$\times \int d t \frac{\left.\left(\left[{ }^{q p} \bar{\eta}_{3}(\tau) \stackrel{\circ}{h}_{3}\right)^{-1 / 4}\right]^{*}\right)^{2}}{\left|\int d t^{q p} \bar{\Psi}(\tau)\left({ }^{q p} \bar{\eta}_{3}(\tau) \stackrel{\circ}{h}_{3}\right)^{*}\right|} ;$

$\bar{\eta}_{k}^{4}(\tau)=\frac{\partial_{i} \int d t^{q p} \bar{¥}(\tau)\left({ }^{q p} \bar{\eta}_{3}(\tau) \stackrel{\circ}{h}_{3}\right)^{*}}{\overline{\mathfrak{w}}_{i} q p \bar{¥}(\tau)\left(q p \bar{\eta}_{3}(\tau) \stackrel{\circ}{h}_{3}\right)^{*}}$,

where ${ }_{1} n_{k}\left(\tau, x^{i}\right)$ and ${ }_{2} n_{k}\left(\tau, x^{i}\right)$ are integration functions. In formulas (56), there are considered nonlinear generat- ing functionals ${ }^{q p} \bar{\Phi}(\tau)={ }^{q p} \bar{\Phi}\left(\tau, x^{i}, t\right)={ }^{q p} \bar{\Phi}\left[{ }^{f l} \bar{\Phi},{ }^{m}\right.$ $\left.\bar{\Phi}, F \bar{\Phi},{ }_{0}^{i n t} \bar{\Phi},{ }_{0}^{\chi} \bar{\Phi}\right]$ characterized by nonlinear symmetries (35) for some prescribed families of nonlinear functionals

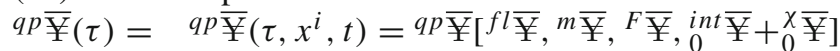
and ${ }^{q p} \bar{\Lambda}(\tau)$. Instead of nonlinear superpositions ( ${ }^{q p} \bar{\Phi}(\tau)$, $\left.q p \bar{¥}(\tau),{ }^{q p} \bar{\Lambda}(\tau)\right)$, we can consider additive data $\left({ }^{a} \bar{\Phi}(\tau)\right.$, $\left.{ }^{a} \bar{¥}(\tau),{ }^{a} \bar{\Lambda}(\tau)\right)$. We can define also general nonlinear, ${ }^{q p} \bar{\eta}_{3}\left(\tau, x^{i}, t\right)$, or additive functionals, ${ }^{a} \bar{\eta}_{3}\left(\tau, x^{i}, t\right)$, for other types polarization functions,

$$
\begin{gathered}
{ }^{q p} \bar{\Phi}^{2}(\tau)=\left.4\right|^{q p} \bar{\Lambda}(\tau)\left[h_{3}^{[0]}(\tau)-{ }^{q p} \bar{\eta}_{3}\left(\tau, x^{i}, t\right) \stackrel{\circ}{h}_{3}\left(x^{k}, t\right)\right] \mid, \\
\left({ }^{q p} \bar{\Psi}^{2}\right)^{*}(\tau)=-\int d t^{q p} \bar{¥}(\tau)\left[{ }^{q p} \bar{\eta}_{3}\left(\tau, x^{i}, t\right) \overline{\dot{h}}_{3}\left(x^{i}, t\right)\right]^{*} .
\end{gathered}
$$

There are defined LC-configurations with zero torsion for target off-diagonal cosmological metrics (40) if there are considered integrable polarization functions

$\bar{\eta}_{i}(\tau)=e^{\psi\left(\tau, x^{i}\right)} / \stackrel{\circ}{g}_{i} ;$ generating function

$\overline{\check{\eta}}_{3}(\tau)={ }^{q p} \overline{\check{\eta}}_{3}\left(\tau, x^{i}, t\right)$;

$\bar{\eta}_{4}(\tau)=-\frac{4\left[\left(\left.\left.\right|^{q p} \overline{\grave{\eta}}_{3}(\tau) \stackrel{\circ}{h}_{3}\right|^{1 / 2}\right)^{*}\right]^{2}}{\grave{h}_{4}\left|\int d t^{q p} \overline{\dddot{Y}}(\tau)\left(q p \overline{\check{\eta}}_{3}(\tau) \stackrel{\circ}{h}_{3}\right)^{*}\right|} ;$

$\bar{\eta}_{k}^{3}(\tau)=\left(\partial_{k} \bar{n}\left(\tau, x^{i}\right)\right) / \stackrel{\circ}{n}_{k} ; \bar{\eta}_{k}^{4}(\tau)=\partial_{k} \bar{A}\left(\tau, x^{i}, t\right) / \stackrel{\circ}{w}_{k}$.

Finally, it should be noted that there is duality on $y^{3}$ and $y^{4}$ coordinates and respective $\mathrm{N}$-connection coefficients for the class of cosmological solutions (56) and the stationary solutions which can be generated via transforms (33).

\section{Conclusions and discussion}

In this article we elaborate a geometric approach to E. Verlinde conjecture $[6,7]$ that gravity can be considered as emergent phenomena of a conventional spacetime elasticity determined by certain entropic forces. We argue that rigorous thermodynamic formulations exist for the models of "entropic spacetime and gravity" which can be derived from generalizations of the Poincaré-Thurston conjecture extended to relativistic geometric flow evolution theories. This is possible if corresponding nonholonomic modifications of Perelman's (entropic type) functionals are performed. For self-similar configurations, certain type entropic flow evolution equations result into corresponding nonholonomic Ricci soliton equations which are equivalent to the motion equations in emergent modified gravity theories, MGTs, and (for certain conditions) in general relativity, GR.

To study geometric flow evolution of Riemannian metrics G. Perelman introduced two Lyapunov type functionals (F- and W-entropy) [45] which was a very important step to the proof of the Poincaré conjecture and elaborating geometric and statistical thermodynamics models for Ricci flows and possible applications in modern physics. 
In a series of our works [34-41,78], we considered various modifications of the $\mathrm{F}$ - and W-entropy functionals for constructing (non) commutative and/or supersymmetric geometric evolution models; investigating nonlinear (fractional and/or locally anisotropic) stochastic and kinetic processes in curved spaces; and elaborating on cosmological scenarios encoding memory and quantum interactions of gravitational and (effective) matter fields. Here we note that the concepts of complex manifolds, supermanifolds and noncommutative geometry, are defined by geometric constructions with nonholonomic distributions on curved spaces. In such a general context, for various entropic spacetime and emergent gravity models, and in geometric flow evolution theories with nonholonomic constraints, we have to develop an unified geometric formalism for metric-affine spaces, generalized Finsler-Lagrange-Hamilton geometry, almost Kähler and noncommutative geometries etc. Such constructions were performed for Hořava-Lifshits, $f(R), R^{2}$, and other types MGTs (see reviews $[68-70,79,80]$ ) with developments for models of thermodynamic/entropic/entanglement and emergent gravity [4-7,16,17,19-25,31,65-67]; and for locally anisotropic kinetic and thermodynamic theories on curved spaces (see $[38,71,78,81-83]$ and references therein) etc.

We have found that using certain classes of nonholonomic variables the (relativistic) geometric flow evolution and Ricci soliton equations, and related motion equations in entropic and other type MGTs, can be decoupled and integrated in some very general forms. This allows us to construct various classes of exact and parametric solutions with generic off-diagonal metrics and generalized connections. The coefficients of new classes of stationary and (in general, locally anisotropic and inhomogeneous) cosmological solutions depend on all spacetime and associated phase space (kinetic and/or thermodynamic) coordinates via generating and integration functions and various types of commutative and noncommutative parameters and integration and physical constants. Such geometric and analytic techniques of constructing exact solutions in geometric flow evolution and MGTs have been developed in the framework of the so-called anholonomic frame deformation method, AFDM [55-57]. For details, examples of exact solutions, and various applications, we cite $[33,38-41,41,58-60,63]$ and references therein.

It should be noted that there were not elaborated corresponding topological methods and a well-defined analytic formalism for investigating geometric evolution equations of metrics of pseudo-Euclidean signature. A number of such conceptual and fundamental issues in nonlinear functional analysis and the geometry of Lorentz manifolds have not addressed or solved by mathematicians. The standard geometric flow paradigm was proposed as the HamiltonPerelman program for Riemannian metrics defining entropy type functionals and deriving nonlinear evolution equations.
To elaborate realistic relativistic physical models we deal with Ricci tensors which in the limit of weak gravitational/elastic flows approximate to the d'Alembert (wave) operator and not to the Laplace (diffusion) one used for Euclidean signatures. So, the original approach to the topology and geometric flows of Riemannian metrics has to be generalized in certain relativistic and nonholonomic forms which are compatible with modern experimental particle physics data and observational cosmology. The PoincaréThurston conjecture can be formulated and proven for nonrelativistic evolution of any 3-d space like hypersurface. In this and partner [32] works, we advocate that using E. Verlinde conjecture on elastic emergent gravity, we can elaborate on generalizations of nonholonomic Ricci flow theories as certain models of relativistic flow evolution. Such models are determined by extensions of Perelman's functionals for 3-d Riemannian metrics to certain modified 4-d Fand W-entropy nonholonomic analogs which are extended on a time like coordinate and/or a temperature like evolution parameter. For certain approaches with rich gravitational vacuum structure, we work with geometric relativistic kinetic/hydrodynamic/thermodynamic models (see details in [38]) or (for instance, in this work) with a $\tau$-parametric theory describing geometric entropic flows determined by relativistic and elastically spacetime modified nonholonomic F- and W-functionals.

As it was mentioned above, there is not yet formulated a rigorous mathematical approach to the theory of relativistic geometric/entropic flows of metrics with Lorentz signature and generalized connections. Nevertheless, we shown that such theories are characterized by certain classes of generalized R. Hamilton equations with effective parametric sources which may encode entropic and quasiperiodic structures (these are necessary for explaining, for instance, the complex structure of dark matter and energy in modern cosmology). Using the AFDM, we proved that such geometric flow evolution equations and their Ricci soliton variants can be decoupled and integrated in very general forms. In Sect. 5, we constructed and analyzed possible physical implications of respective entropic locally anisotropic cosmological solutions. A series of our former results on astrophysical and cosmological models with quasiperiodic, pattern forming, quasicrystal time like structures, see [59-64] and references therein, were used for developing in this work similar models for the entropic geometric flow and gravity theories. Such exact and parametric solutions provide also explicit examples of entropic gravity models developed in a phenomenological manner in $[6,7,65]$. So, we conclude that the E. Verlinde conjecture on entropic character of gravity can be related to a relativistic extension of the Poincaré-Thurston conjecture. Even such geometric ideas have not been proven as explicit theorems in modern geometric analysis, there are rigorous exact solutions of respective systems of nonlinear 
PDES which support such entropic gravity and flow evolution ideas.

In Sects. 4 and 5, we shown how to construct in explicit form entropic and quasiperiodic solutions for relativistic geometric flows, nonholonomic Ricci solitons and generalized gravitational field equations. Such a techniques was elaborated similarly to MGTs with quasiperiodic structure (constructed and studied in this and our partner works [32, 63, 64]) and involves generic off-diagonal metric and nonholonomically deformed non-Riemannian linear and nonlinear connections. We emphasized, see also [41], that such configurations are not characterized, in general, by certain entropyarea, holographic or duality conditions. As a consequence, it is not possible to elaborate on thermodynamic models of entropic MGTs and physical properties of their exact or parametric solutions using only the concepts related to the Bekenstein-Hawking entropy. We consider that there is an alternative and more general way when stationary and cosmological solutions in geometric/entropic flow evolution theories, MGTs and GR, can be defined and characterized by nonholonomic deformations of Perelman's W-entropy. Such constructions are similar to the well-known results on relativistic locally anisotropic thermodynamics and kinetics $[38,71]$ and can be generalized for emergent classical and quantum gravity theories.

Finally, we note that our geometric/entropic flow approach to MGTs provides new mathematical methods and applications in the theory of classical and quantum informatics, for research of quantum systems with entanglement, models of quantum and emergent gravity, and accelerating cosmology and dark energy/matter interactions etc. Our further research programs are related to developments in such directions.

Acknowledgements This research develops authors' former research programs on geometric flows and applications in physics and information theory partially supported by a fellowship at IMAFF CSIC Madrid; a visit at Fields Institute, Toronto; a project IDEI, PN-II-ID-PCE-20113-0256; fellowships at CERN and DAAD programs for W. Heisenberg (M. Plank) Institute, Munich.

Data Availability Statement This manuscript has no associated data or the data will not be deposited. [Authors' comment: Associated data were not considered for this work.]

Open Access This article is licensed under a Creative Commons Attribution 4.0 International License, which permits use, sharing, adaptation, distribution and reproduction in any medium or format, as long as you give appropriate credit to the original author(s) and the source, provide a link to the Creative Commons licence, and indicate if changes were made. The images or other third party material in this article are included in the article's Creative Commons licence, unless indicated otherwise in a credit line to the material. If material is not included in the article's Creative Commons licence and your intended use is not permitted by statutory regulation or exceeds the permitted use, you will need to obtain permission directly from the copyright holder. To view a copy of this licence, visit http://creativecomm ons.org/licenses/by/4.0/.

Funded by SCOAP ${ }^{3}$.

\section{References}

1. J.D. Bekenstein, Black holes and the second law. Nuovo Cimento Lett. 4, 737-740 (1972)

2. J.D. Bekenstein, Black holes and entropy. Phys. Rev. D 7, 2333 2346 (1973)

3. J.M. Bardeen, B. Carter, S.W. Hawking, The four laws of black hole mechanics. Commun. Math. Phys. 31, 161 (1973)

4. T. Jacobson, Entanglement equilibrium and the Einstein equation. Phys. Rev. Lett. 116, 201101 (2016)

5. T. Padmanabhan, Theromdynamical aspects of gravity: new insights. Rep. Prog. Phys. 73, 046901 (2010)

6. E.P. Verlinde, On the origin of gravity and the laws of Newton. JHEP 1104, 029 (2011)

7. E.P. Verlinde, Emergent gravity and the dark universe. SciPost Phys. 2(3), 016 (2017)

8. A. Strominger, C. Vafa, Microscopic origin of the BekensteinHawking entropy. Phys. Lett. B 379, 99 (1996)

9. L. Susskind, The world as a hologram. J. Math. Phys. 36, 63776396 (1995)

10. G. 't Hooft, Dimensional reduction in quantum gravity. arXiv:gr-qc/9310026

11. L. Susskind, L. Thorlacius, J. Uglum, The stretched horizon and black hole complementarity. Phys. Rev. D 48, 3743-3761 (1993)

12. E. Witten, Anti-de Sitter space and holography. Adv. Theor. Math. Phys. 2, 253-291 (1998)

13. O. Aharaony, S.S. Gubser, J.M. Maldacena, H. Ooguri, Y. Oz, Large- $\mathrm{N}$ field theories, string theory and gravity. Phys. Rep. 323, 183-386 (2000)

14. J.M. Maldacena, The large $\mathrm{N}$ limit of superconformal field theories and supergravity. Adv. Theor. Math. Phys. 2, 231 (1998)

15. A. Ashtekar, B. Krishnan, Isolated and dynamical horizons and their application. Living Rev. Relativ. 7, 10 (2004)

16. M. Van Raamsdonk, Building up spacetime with quantum entanglement. Gen. Relativ. Gravit. 42, 2323 (2010). arXiv:1005.3035

17. M. Van Raamsdonk, Building up spacetime with quantum entanglement. Int. J. Mod. Phys. D 19, 2429 (2010)

18. J. Maldacena, L. Susskind, Cool horizons for entangled black holes. Fortschr. Phys. 61, 781 (2013)

19. N. Lashkari, M.B. McDermott, M. Van Raamsdonk, Gravitational dynamics from entanglement 'thermodynamics'. JHEP 1404, 195 (2014)

20. S. Ryu, T. Takayanagi, Holographic derivation of entanglement entropy from AdS/CFT. Phys. Rev. Lett. 96, 181602 (2006)

21. S. Ryu, T. Takayanagi, Aspects of holographic entanglement entropy. JHEP 1105, 036 (2011)

22. S. Lloyd, The quantum geometric limit. arXiv:1206.6559

23. T. Faulkner, M. Guica, T. Harman, R.C. Myers, M. Van Raamsdonk, Gravitation from entanglement and holographic CFTs. JHEP 1403, 051 (2015)

24. B. Swingle, Entanglement renormalization and holography. Phys. Rev. D 86, 065007 (2012)

25. F. Pastawski, B. Yoshida, D. Harlow, J. Preskill, Holographic quantum error-correcting codes: toy models for the bulk/boundary correspondence. JHEP 1506, 149 (2015)

26. E. Oh, I.Y. Park, S.-J. Sin, Complete Einstein equation from the generalized first law of entanglement. Phys. Rev. D 98, 026020 (2018)

27. E. Witten, A mini-introduction to information theory. Riv. Nuovo Cim. 43, 187-227 (2020)

28. J. Preskill, Lecture notes. http://www.theory.caltech.edu/ preskill/ ph219/index.html\#lecture

29. H. Casini, M. Huerta, R.C. Myers, Towards a derivation of holographic entanglement entropy. JHEP 1105, 036 (2011) 
30. A. Lewkowycz, J. Maldacena, Generalized gravitational entropy. JHEP 1308, 090 (2013)

31. S.N. Solodukhin, Entanglement entropy of black holes. Living Rev. Relativ. 14, 8 (2011)

32. I. Bubuianu, S. Vacaru, E.V. Veliev, Entropy functionals and thermodynamics of relativistic geometric flows, stationary quasiperiodic Ricci solitons, and gravity. Ann. Phys. 423, 168333 (2020)

33. S. Vacaru, Ricci flows and solitonic pp-waves. Int. J. Mod. Phys. A 21, 4899-4912 (2006)

34. S. Vacaru, Nonholonomic Ricci flows: II. Evolution equations and dynamics. J. Math. Phys. 49, 043504 (2008)

35. S. Vacaru, Spectral functionals, nonholonomic Dirac operators, and noncommutative Ricci flows. J. Math. Phys. 50, 073503 (2009)

36. S. Vacaru, Fractional nonholonomic Ricci flows. Chaos Solitons Fractals 45, 1266-1276 (2012)

37. S. Vacaru, Almost Kaehler Ricci flows and Einstein and LagrangeFinsler structures on Lie algebroids. Mediterr. J. Math. 12, 13971427 (2015)

38. V. Ruchin, O. Vacaru, S. Vacaru, Perelman's W-entropy and statistical and relativistic thermodynamic description of gravitational fields. Eur. Phys. J. C 77, 184 (2017)

39. T. Gheorghiu, V. Ruchin, O. Vacaru, S. Vacaru, Geometric flows and Perelman's thermodynamics for black ellipsoids in R2 and Einstein gravity theories. Ann. Phys. 369, 1-35 (2016)

40. S. Rajpoot, S. Vacaru, On supersymmetric geometric flows and R2 inflation from scale invariant supergravity. Ann. Phys. 384, 20-60 (2017)

41. L. Bubuianu, S. Vacaru, Black holes with MDRs and BekensteinHawking and Perelman entropies for Finsler-Lagrange-Hamilton spaces. Ann. Phys. 404, 10-38 (2019)

42. R.S. Hamilton, Three-manifolds with positive Ricci curvature. J. Differ. Geom. 17, 255-306 (1982)

43. R.S. Hamilton, The Ricci flow on surfaces, in Mathematics and General Relativity. Contemporary Mathematics, vol. 71 (American Mathematical Society, Providence, 1988), pp. 237-262

44. R.S. Hamilton, in Surveys in Differential Geometry, vol. 2 (International Press, Vienna, 1995), pp. 7-136

45. G. Perelman, The entropy formula for the Ricci flow and its geometric applications. arXiv:math.DG/0211159

46. H.-D. Cao, H.-P. Zhu, A complete proof of the Poincaré and geometrization conjectures-application of the HamiltonPerelman theory of the Ricci flow. Asian J. Math. 10, 165-495 (2006)

47. J.W. Morgan, G. Tian, Ricci Flow and the Poincaré Conjecture, AMS, Clay Mathematics Monographs, vol. 3 (2007)

48. B. Kleiner, J. Lott, Notes on Perelman's papers. Geom. Topol. 12, 2587-2855 (2008)

49. D. Friedan, Nonlinear models in $2+\varepsilon$ dimensions. PhD Thesis (Berkely) LBL-11517, UMI-81-13038, Aug 1980 (1980)

50. D. Friedan, Nonlinear models in $2+\varepsilon$ dimensions. Phys. Rev. Lett. 45, 1057-1060 (1980)

51. D. Friedan, Nonlinear models in $2+\varepsilon$ dimensions. Ann. Phys. 163, 318-419 (1985)

52. E.P. Verlinde, H.L. Verlinde, RG flow, gravity and the cosmological constant. JHEP 0005, 034 (2000)

53. A.A. Tseytlin, On sigma model RG flow, "central charge" action and Perelman's entropy. Phys. Rev. D 75, 064024 (2007)

54. S. Jackson, R. Pourhasan, H. Verlinde, Geometric RG flow. arXiv: 1312.6914

55. S. Vacaru, Exact solutions with noncommutative symmetries in Einstein and gauge gravity. J. Math. Phys. 46, 042503 (2005)

56. S. Vacaru, Nonholonomic Ricci flows, exact solutions in gravity, and symmetric and nonsymmetric metrics. Int. J. Theor. Phys. 48, 579-606 (2009)

57. S. Vacaru, On general solutions in Einstein gravity. Int. J. Geom. Methods Mod. Phys. 8, 9-21 (2011)
58. T. Gheorghiu, O. Vacaru, S. Vacaru, Off-diagonal deformations of Kerr black holes in Einstein and modified massive gravity and higher dimensions. Eur. Phys. J. C 74, 3152 (2014)

59. L. Bubuianu, K. Irwin, S. Vacaru, Heterotic supergravity with internal almost-Kaehler spaces; instantons for $\mathrm{SO}(32)$, or $\mathrm{E} 8 \times \mathrm{E} 8$, gauge groups; and deformed black holes with soliton, quasiperiodic and/or pattern-forming structures. Class. Quantum Gravity 34, $075012(2017)$

60. S. Vacaru, Off-diagonal ekpyrotic scenarios and equivalence of modified, massive and/or Einstein gravity. Phys. Lett. B 752, 27 33 (2016)

61. M.M. Amaral, R. Ashheim, L. Bubuianu, K. Irwin, S.I. Vacaru, D. Woolridge, Anamorphic quasiperiodic universes in modified and Einstein gravity with loop quantum gravity corrections. Class. Quantum Gravity 34, 185002 (2017)

62. R. Aschheim, L. Bubuianu, F. Fang, K. Irwin, V. Ruchin, S. Vacaru, Starobinsky inflation and dark energy and dark matter effects from quasicrystal like spacetime structure. Ann. Phys. 394, 120-138 (2018)

63. L. Bubuianu, S. Vacaru, Deforming black hole and cosmological solutions by quasiperiodic and/or pattern forming structures in modified and Einstein gravity. Eur. Phys. J. C 78, 393 (2018)

64. S. Vacaru, Space-time quasicrystal structures and inflationary and late time evolution dynamics in accelerating cosmology. Class. Quantum Gravity 35, 245009 (2018)

65. S. Hossenfelder, Covariant version of Verlinde's emergent gravity. Phys. Rev. D 95, 124018 (2017)

66. D.-C. Dai, D. Stojkovic, Comment on 'Covariant version of Verlinde's emergent gravity'. Phys. Rev. D 96, 108501 (2017)

67. D.-C. Dai, D. Stojkovic, Inconsistencies in Verlinde's emergent gravity. JHEP 1711, 007 (2017)

68. S. Nojiri, S.D. Odintsov, V.K. Oikonomou, Modified gravity theories in nutshell: inflation, bounce and late-time evolution. Phys. Rep. 692, 1-104 (2017)

69. S. Capozziello, V. Faraoni, Beyond Einstein Gravity (Springer, Berlin, 2010)

70. M. Wali Hossain, R. Myrzakulov, M. Sami, E.N. Saridakis, Unification of inflation and drak energy á la quintessential inflation. Int. J. Mod. Phys. D 24, 1530014 (2015)

71. S. Vacaru, Locally anisotropic kinetic processes and thermodynamics in curved spaces. Ann. Phys. (N.Y.) 290, 83-123 (2001)

72. C.W. Misner, K.S. Thorne, J.A. Wheeler, Gravitation (Freeman, New York, 1973)

73. P. Das, S. Pan, S. Ghosh, Thermodynamics and phase transition in Shapere-Wilczek fgh model: cosmological time crystal in quadratic gravity. Phys. Lett. B (accepted). arXiv:1810.06606

74. A. Shapere, F. Wilczek, Classical time crystals. Phys. Rev. Lett. 109, $160402(2012)$

75. F. Wilczek, Quantum time crystals. Phys. Rev. Lett. 109, 160401 (2012)

76. F. Wilczek, Wilczek reply. Phys. Rev. Lett. 110, 118902 (2013)

77. A.D. Shapere, F. Wilczek, Realization of "time crystal" Lagrangians and emergent sisyphus dynamics. arXiv:1708.3348

78. S. Vacaru, Nonholonomic relativistic diffusion and exact solutions for stochastic Einstein spaces. Eur. Phys. J. P 127, 32 (2012)

79. S. Basilakos, A.P. Kouretsis, E.N. Saridakis, P. Stavrinos, Resembling dark energy and modified gravity with Finsler-Randers cosmology. Phys. Rev. D 83, 123510 (2013)

80. T. Elghozi, N.E. Mavromatos, M. Sakellariadou, M.F. Yusaf, The D-material universe. JCAP 02, 60 (2016)

81. G. Ruppeiner, Riemannian geometry in thermodynamic fluctuation theory. Rev. Mod. Phys. 67, 605-659 (1995) [Erratum: 68, 313 (1996)] 
82. H. Quevedo, Geometrothermodynamics. J. Math. Phys. 48, 013506 (2007)
83. C. Castro Perelman, Thermal relativity, corrections of black hole entropy, Born's reciprocal relativity theory and quantum gravity. Can J. Phys. 97, 1309-1316 (2019) 